\title{
Inelastic scattering of quasiparticles in a superconductor with magnetic impurities
}

\author{
A. G. Kozorezov, ${ }^{1}$ A. A. Golubov, ${ }^{2}$ J. K. Wigmore, ${ }^{1}$ D. Martin, ${ }^{3}$ P. Verhoeve, ${ }^{3}$ R. A. Hijmering, ${ }^{3}$ and I. Jerjen ${ }^{3}$ \\ ${ }^{1}$ Department of Physics, Lancaster University, Lancaster LA1 4YB, United Kingdom \\ ${ }^{2}$ Department of Applied Physics, University of Twente, P.O. Box 217, 7500 AE Enschede, The Netherlands \\ ${ }^{3}$ Advanced Studies and Technology Preparation Division, Directorate of Science and Robotic Exploration of the European Space Agency, \\ ESTEC, Noordwijk, The Netherlands
}

(Received 29 July 2008; revised manuscript received 15 September 2008; published 3 November 2008)

\begin{abstract}
We show that inelastic scattering of quasiparticles by trace concentrations of magnetic impurities may result in significant changes in the nonequilibrium properties of superconductors. We used the approach of MüllerHartmann and Zittartz to model Kondo scattering of conduction electrons by the magnetic impurities, and hence, to calculate the rates of (i) quasiparticle trapping into the localized impurity states, (ii) trap-enhanced recombination, (iii) pair breaking, and (iv) detrapping of localized quasiparticles by phonons, including both deformation-potential and spin-lattice couplings. Our results indicate that these processes will give rise to anomalies in the temperature dependence of kinetic parameters, which should be easily observable.
\end{abstract}

DOI: 10.1103/PhysRevB.78.174501

PACS number(s): 74.25.Fy, 72.10.Fk, 74.40.+k

\section{INTRODUCTION}

The study of magnetic impurities in superconductors originated with the pioneering work by Abrikosov and Gor' kov $^{1}$ in 1961, who demonstrated that the primary effect is the destruction of superconducting coherence, while a further consequence was predicted to be the formation of intragap quasiparticle $(\mathrm{QP})$ states localized on the impurity atoms themselves. ${ }^{2-8}$ Over the following three decades the global consequences of magnetic disorder, such as the modification of the superconducting energy gap and coherence length, were studied widely. However interest in the possible localization of QP states remained relatively dormant until the topic was stimulated by the ground-breaking experiments of Yazdani et $a l .{ }^{9}$ In this work the technique of scanning tunneling microscopy was used to map directly the spatial distribution of QP states due to adatoms and to chemically introduced impurities. The results confirmed decisively the predicted localization for both conventional and unconventional superconductors. A comprehensive review of recent developments in the understanding of impurity-induced states in superconductors was given by Balatsky et al. ${ }^{10}$

To date, however, little attention has been paid to the role which these localized states may play in the evolution of nonequilibrium excitations in a superconductor, for example, to the potential effects of the trapping of QPs by localized states and to the recombination of one mobile with one localized QP, which might also be expected in analogy with the role of deep levels for electrons and holes in semiconductors. In this paper we present calculations which show that both deformation-potential and spin-lattice couplings play a significant role in facilitating transitions between the continuum and discrete states bound to impurities. Thus QPs initially in continuum states may undergo inelastic scattering with phonon emission and become localized at impurity atoms. The impurities may also act as recombination centers and provide rapid thermalization of a nonequilibrium initial distribution. Furthermore, the formation of an intragap band of impurity levels deep inside the gap, potentially even overlapping the ground state, will modify the temperature dependence of thermalization. Finally, activation of localized QPs into the continuum may result in an anomalous temperature dependence of observable parameters characterizing the nonequilibrium state, such as QP lifetime. ${ }^{11}$

It might be expected that any such effects of isolated magnetic impurities in superconductors, while being of significant academic interest, could hardly influence the macroscopic properties of a nominally pure device or sample. However, an important conclusion from our calculations is that the QP processes described above show up at much lower impurity concentrations than has been previously realized. We believe that experimental results already published provide clear evidence for the involvement of the processes considered in this paper. Indeed the original motivation for the work was to understand unexplained results from experiments carried out by ourselves and other groups on nonequilibrium transport properties of nominally pure superconductors at very low temperatures. Anomalous experimental data were obtained for quasiparticle lifetimes which (in some cases) were orders of magnitude shorter than would be expected for pure material. The results and their reinterpretation will be described in a later section. The conclusion of the present paper is that the likely cause of such effects is the inelastic scattering of quasiparticles by the discrete states localized on magnetic impurities. Our calculations confirm that magnetic impurities at concentrations of only few ppm can give rise to quasiparticle trapping, enhanced recombination, and pair breaking which at low temperatures totally dominate intrinsic effects.

\section{MODEL}

To describe inelastic scattering of QPs in a superconductor with magnetic impurities, we will consider the bound states within the model originally developed by MüllerHartmann and Zittartz ${ }^{7,8}$ for quantum spins in a fully gapped superconductor. Within this model two bound states exist which are symmetrically located with respect to the center of the energy gap. For ferromagnetic coupling, and for antiferromagnetic coupling with Kondo temperature $\left(T_{K}\right)$ either 
very small or very large compared to the superconducting transition temperature $\left(T_{c 0}\right)$ of the intrinsic superconductor (without magnetic impurities), these bound states lie very close to the gap edges. However, for $T_{K} \sim T_{c 0}$ the bound states move close to the center of the gap. We will consider the most general case of arbitrary location of the discrete levels inside the superconductor gap.

In the model of Müller-Hartmann and Zittartz the Hamiltonian of the system takes the form

$$
H=H_{0}+H^{\prime},
$$

where $H_{0}$ is the Hamiltonian of an intrinsic BCS superconductor and $H^{\prime}$ describes the interaction between impurity atoms and conduction electrons. The corresponding interaction potential has the form ${ }^{1}$

$$
v(\mathbf{r})=\sum_{i}\left[u_{1}\left(\mathbf{r}-\mathbf{R}_{i}\right)+u_{2}\left(\mathbf{r}-\mathbf{R}_{i}\right) \sigma \cdot S\right]
$$

where $\mathbf{R}_{i}$ is the coordinate of the impurity atom, $S$ is its spin, and $\sigma_{\alpha \beta}^{s}$ are the spin Pauli matrices. Here the first term describes the spin independent part of the impurity scattering potential and the second term describes the exchange interaction.

To consider phonon-assisted electronic transitions involving the bound states, we need terms describing the electronphonon interaction through both the deformation-potential and the spin-lattice interactions. We use the four-dimensional matrix formalism so that all quantities of interest are fourcomponent operators or $4 \times 4$ matrices. The full interaction Hamiltonian describing phonon-assisted electronic transitions has the form

$$
H_{\mathrm{int}}=\frac{1}{2} \int d \mathbf{r} \psi^{+}(\mathbf{r}) V(\mathbf{r}) \psi(\mathbf{r}),
$$

where $\psi(\mathbf{r})$ and $\psi^{+}(\mathbf{r})$ are four-component operators,

$$
\psi(\mathbf{r})=\left(\begin{array}{c}
\psi_{\uparrow}(\mathbf{r}) \\
\psi_{\downarrow}(\mathbf{r}) \\
\psi_{\uparrow}^{+}(\mathbf{r}) \\
\psi_{\downarrow}^{+}(\mathbf{r})
\end{array}\right), \quad \psi^{+}(\mathbf{r})=\left[\psi_{\uparrow}^{+}(\mathbf{r}), \psi_{\downarrow}^{+}(\mathbf{r}), \psi_{\uparrow}(\mathbf{r}), \psi_{\downarrow}(\mathbf{r})\right],
$$

$V(\mathbf{r})=\Sigma_{i} \mathbf{Q}_{i} \cdot \nabla U(\mathbf{r})$, and $U(\mathbf{r})$ is the $4 \times 4$ matrix of the form

$$
U(\mathbf{r})=\left(\begin{array}{cc}
u_{1}(\mathbf{r}) \sigma_{0}+u_{2}(\mathbf{r}) \sigma \cdot S & 0 \\
0 & -\left[u_{1}(\mathbf{r}) \sigma_{0}+u_{2}(\mathbf{r}) \sigma \cdot S\right]^{\operatorname{tr}}
\end{array}\right),
$$

where the superscript denotes the transposed matrix, and $Q_{i}$ is the lattice displacement of the impurity due to vibrations. This expression has been derived by expanding the first and the second terms in Eq. (2), respectively, to include the displacement of the impurity atom from its equilibrium site.

\section{SELF-ENERGY}

In a superconductor described by Hamiltonian (1) for an impurity with either ferromagnetic or antiferromagnetic ex- change, bound states that split off from the gap are formed. ${ }^{7}$ We will consider the dilute impurity limit $c \ll 1$. Here $c$ is the dimensionless impurity density in units of the condensate density $2 N(0) \Delta$, where $N(0)$ is the density of states at the Fermi level per spin in the normal state and $\Delta$ is the gap. This Hamiltonian describes the unperturbed system while the interaction described by Hamiltonian (3) causes inelastic transitions between the continuum and discrete electronic states. The usual electron-phonon interaction, which leads to transitions in the continuum part of the spectrum of electronic excitations in a superconductor, has not been included because the effect of magnetic impurities on electron-phonon interactions under these conditions is small. Our derivation of the transition rates follows the Eliashberg formulation. ${ }^{12,13}$ In the dilute limit the shifts of the gap edge and all levels in the continuum spectrum remain small, being proportional to the density of impurities. Therefore we disregard all effects of magnetic impurities on the continuum QP spectrum-both level shift and broadening. It is important to note that although the Kondo impurity affects electron spin, however, scattering remains elastic: energies of spin-up and spin-down impurity states are identical. Our objective is to discuss inelastic-scattering events. Therefore, we will determine the spatially averaged single-particle Green's function for the continuum spectrum with the additional interaction channel given by Eq. (3). The extra contribution to the imaginary part of the poles of this Green's function will describe the rates of transition in which we are interested.

We will derive the expression for the extra contribution to the self-energy of the QP in a continuum spectrum due to interaction with discrete levels. By neglecting the scattering on different impurities, which gives the terms of the order of $c^{2} \ll 1$, and separating the statistical averages over electron and phonon operators, we obtain the Green's function in the form

$$
\begin{aligned}
G\left(\mathbf{x}, \mathbf{x}^{\prime}\right)= & G_{0}^{m}\left(\mathbf{x}, \mathbf{x}^{\prime}\right)+\sum_{a} \int d \mathbf{x}_{1} d \mathbf{x}_{2} G_{0}^{m}\left(\mathbf{x}, \mathbf{x}_{1}\right) \\
& \times \mathbf{Q}_{a} \cdot \nabla U\left(\mathbf{x}_{1}-\mathbf{R}_{a}\right) G_{0}^{m}\left(\mathbf{x}_{1}, \mathbf{x}_{2}\right) \\
& \times \mathbf{Q}_{a} \cdot \nabla U\left(\mathbf{x}_{2}-\mathbf{R}_{a}\right) G_{0}^{m}\left(\mathbf{x}_{2}, \mathbf{x}^{\prime}\right)+\ldots
\end{aligned}
$$

Here $G_{0}^{m}\left(\mathbf{x}, \mathbf{x}_{1}\right)$ is the unperturbed Green's function for the system described by Hamiltonian (1). ${ }^{7}$ In the limit $c \ll 1$ spatial averaging can be carried out separately for all elements of the Dyson equation. Indeed, we may replace the external Green's functions $G_{0}^{m}\left(\mathbf{x}, \mathbf{x}_{1}\right)$ and $G_{0}^{m}\left(\mathbf{x}_{2}, \mathbf{x}^{\prime}\right)$ by the spatially averaged Green's functions $\bar{G}_{0}^{m}\left(\mathbf{x}-\mathbf{x}_{1}\right)$ and $\bar{G}_{0}^{m}\left(\mathbf{x}_{2}-\mathbf{x}^{\prime}\right)$, respectively, where the top bar denotes the procedure of spatial averaging. Since the second term contains the summation over impurities, it is already proportional to impurity density $c \ll 1$; hence, replacing the external Green's functions by spatially averaged ones introduces inaccuracy only in higherorder terms. Thus for the spatially averaged Green's function we obtain 


$$
\bar{G}\left(\mathbf{x}, \mathbf{x}^{\prime}\right)=\bar{G}_{0}^{m}\left(\mathbf{x}, \mathbf{x}^{\prime}\right)+\sum_{a} \int d \mathbf{x}_{1} d \mathbf{x}_{2} \bar{G}_{0}^{m}\left(\mathbf{x}-\mathbf{x}_{1}\right) \overline{\mathbf{Q}_{a} \cdot \nabla U\left(\mathbf{x}_{1}-\mathbf{R}_{a}\right) G_{0}^{m}\left(\mathbf{x}_{1}, \mathbf{x}_{2}\right) \mathbf{Q}_{a} \cdot \nabla U\left(\mathbf{x}_{2}-\mathbf{R}_{a}\right)} \bar{G}_{0}^{m}\left(\mathbf{x}_{2}-\mathbf{x}^{\prime}\right)+\ldots
$$

For the Matsubara Green's function we therefore obtain

$$
\begin{aligned}
\bar{G}\left(\mathbf{x}, \mathbf{x}^{\prime}, \omega_{n}\right)= & \bar{G}_{0}^{m}\left(\mathbf{x}, \mathbf{x}^{\prime}, \omega_{n}\right)-T \sum_{n^{\prime}} \sum_{\mathbf{q} j} \frac{\hbar D_{\mathbf{q}, j}\left(\omega_{n}-\omega_{n^{\prime}}\right)}{2 M N \omega_{\mathbf{q} j}} \int d \mathbf{x}_{1} d \mathbf{x}_{2} \bar{G}_{0}^{m}\left(\mathbf{x}-\mathbf{x}_{1}, \omega_{n}\right) \\
& \times \sum_{a} \overline{\mathbf{e}_{\mathbf{q}, j} \cdot \nabla U\left(\mathbf{x}_{1}-\mathbf{R}_{a}\right) G_{0}^{m}\left(\mathbf{x}_{1}, \mathbf{x}_{2}, \omega_{n^{\prime}}\right) \mathbf{e}_{\mathbf{q} j} \cdot \nabla U\left(\mathbf{R}_{a}-\mathbf{x}_{2}\right)} \bar{G}_{0}^{m}\left(\mathbf{x}_{2}-\mathbf{x}^{\prime}, \omega_{n}\right)+\ldots
\end{aligned}
$$

Here $\omega_{n}$ is the Matsubara frequency with $\omega_{n}=(2 n+1) \pi T, T$ is the temperature, $D_{\mathbf{q}, j}\left(\omega_{n}\right)$ is the phonon Green's function, and $\mathbf{q}, j, \omega_{\mathbf{q}, j}, \mathbf{e}_{\mathbf{q}, j}, M$, and $N$ are phonon wave vector, branch index, phonon frequency, mode polarization vector, mass of the unit cell, and number of cells, respectively. To perform spatial averaging we assume a random distribution of impurities and replace the summation over impurities in Eq. (8) by integration over their coordinates according to $\Sigma_{a}(\ldots) \rightarrow n_{i} \int d \mathbf{x}(\ldots)$, where $n_{i}$ is the impurity density. For $G_{0, n^{\prime}}^{m}\left(\mathbf{x}_{1}, \mathbf{x}_{2}\right)$ we use the explicit form given in ${ }^{7}$

$$
\begin{aligned}
G_{0}^{m}\left(\mathbf{x}_{1}, \mathbf{x}_{2}, \omega_{n}\right)= & G_{0}\left(\mathbf{x}_{1}-\mathbf{x}_{2}, \omega_{n}\right) \\
& +\frac{J}{N} \sum_{i} G_{0}\left(\mathbf{x}_{1}-\mathbf{x}_{i}, \omega_{n}\right) t\left(\omega_{n}\right) G_{0}\left(\mathbf{x}_{i}-\mathbf{x}_{2}, \omega_{n}\right),
\end{aligned}
$$

where $G_{0}\left(\mathbf{x}, \omega_{n}^{\prime}\right)$ is the Green's function of the perfect crystal, $J$ is the exchange integral, and $t\left(\omega_{n^{\prime}}\right)$ is the transfer matrix. ${ }^{7}$ Integrating over the impurity coordinates makes the system translation invariant, leading to the following expression for the spatially averaged Green's function in momentum space:

$$
\bar{G}_{0}^{m}(\mathbf{k})=G_{0}(\mathbf{k})+\frac{N_{i} J}{N} G_{0}(\mathbf{k}) t G_{0}(\mathbf{k}),
$$

where $N_{i}$ is the number of impurity atoms. In momentum space we may rewrite Eq. (8) in the form of the Dyson equation assuming that spatial averaging has already been performed (and from now on omitting top bars),

$$
G^{m}\left(\mathbf{p}, \omega_{n}\right)=G_{0}^{m}\left(\mathbf{p}, \omega_{n}\right)+G_{0}^{m}\left(\mathbf{p}, \omega_{n}\right) \Sigma_{p h}\left(\mathbf{p}, \omega_{n}\right) G^{m}\left(\mathbf{p}, \omega_{n}\right) .
$$

The expression for self-energy $\Sigma_{p h}\left(\mathbf{p}, \omega_{n}\right)$ describing the transitions into discrete states, for which only the second term in Eq. (10) is responsible, can be written as

$$
\begin{aligned}
\Sigma_{p h}\left(\mathbf{p}, \omega_{n}\right)= & -T \sum_{n^{\prime}} \sum_{\mathbf{q} j} \frac{\hbar D_{\mathbf{q}, j}\left(\omega_{n}-\omega_{n^{\prime}}\right)}{2 M N \omega_{\mathbf{q} j}} \\
& \times \int \frac{d \mathbf{k} d \mathbf{k}^{\prime}}{(2 \pi)^{6}}\left(\mathbf{e}_{\mathbf{q} j} \cdot \mathbf{p}-\mathbf{k}\right) U(\mathbf{p}-\mathbf{k}) \\
& \times \frac{J N_{i}}{N} G_{0}\left(\mathbf{k}, \omega_{n^{\prime}}\right) t\left(\omega_{n^{\prime}}\right) G_{0}\left(\mathbf{k}^{\prime}, \omega_{n^{\prime}}\right) U\left(\mathbf{p}-\mathbf{k}^{\prime}\right) \\
& \times\left(\mathbf{e}_{\mathbf{q} j} \cdot \mathbf{p}-\mathbf{k}^{\prime}\right) .
\end{aligned}
$$

Here self-energy, the Green's functions, transfer matrix, and potentials are $4 \times 4$ matrices. For $G_{0}\left(\mathbf{k}, \omega_{n}\right)$ we have

$$
\begin{aligned}
G_{0}\left(\mathbf{k}, \omega_{n}\right) & =\frac{1}{\omega_{n}^{2}+\xi_{\mathbf{k}}^{2}+\Delta^{2}}\left(\begin{array}{cc}
-\left(i \omega_{n}+\xi_{\mathbf{k}}\right) \sigma_{0} & \Delta i \sigma_{2} \\
-\Delta i \sigma_{2} & -\left(i \omega_{n}-\xi_{\mathbf{k}}\right) \sigma_{0}
\end{array}\right) \\
& =-\frac{i \omega_{n} \sigma_{0} \tau_{0}+\xi_{\mathbf{k}} \sigma_{0} \tau_{3}+\Delta \sigma_{2} \tau_{2}}{\omega_{n}^{2}+\xi_{\mathbf{k}}^{2}+\Delta^{2}},
\end{aligned}
$$

where $\sigma$ and $\tau$ are Pauli matrices with $\sigma$ operating in spin space and $\tau$ in the space composed of electron-hole states, with

$$
\sigma_{0}=\left(\begin{array}{ll}
1 & 0 \\
0 & 1
\end{array}\right), \quad \sigma_{2}=\left(\begin{array}{ll}
0 & -i \\
i & 0
\end{array}\right), \quad \sigma_{3}=\left(\begin{array}{ll}
1 & 0 \\
0 & -1
\end{array}\right),
$$

and similarly for $\tau$. The $\sigma \tau$ products in Eq. (13) describe direct products of Pauli matrices leading to $4 \times 4$ matrices. Figure 1 shows the Feynman diagram corresponding to selfenergy (12). Although Fig. 1 looks similar to the usual diagram describing the electron-phonon interaction, it also shows significant differences. Momentum can be transferred to the impurity, and therefore, it is not conserved at vertices, so that $\mathbf{k}$ and $\mathbf{k}^{\prime}$ are independent of $\mathbf{p}$, initial QP momentum,

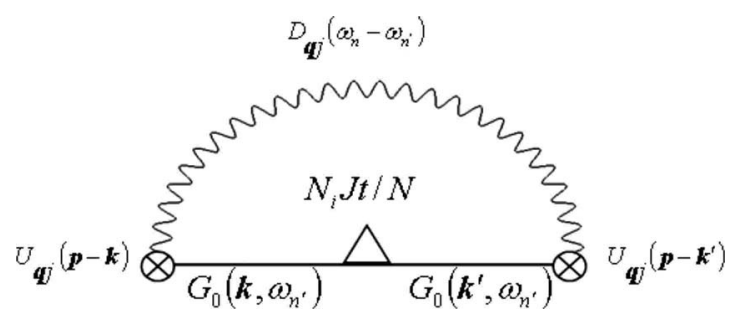

FIG. 1. Feynman diagram for self-energy for electron-phonon interaction with Kondo impurities. 
and $\mathbf{q}$ is the momentum which is carried by the phonon. The triangle in the center of the diagram denotes the interaction with the impurity involving virtual transitions to and from the localized state, which are subject only to energy conservation. The interaction energy (3) contains the gradient term of a function which depends only on the distance to the impurity atom and cancels out if averaged over the whole volume. Therefore we have

$$
\sum_{\mathbf{k}} \mathbf{e}_{\mathbf{q} j} \cdot(\mathbf{p}-\mathbf{k}) U(\mathbf{p}-\mathbf{k})=0
$$

where summation runs over all wave vectors (not limited to the first Brillouin zone). Clearly the cancellation occurs because the function under the sum is an odd function of $\mathbf{p}$ -k to be summed over symmetric limits.

Solving Eq. (9) for the transfer matrix yields

$$
\frac{N_{i} J}{N} t\left(\omega_{n}\right)=G_{0}^{-1}\left(\mathbf{k}, \omega_{n}\right) G_{m}\left(\mathbf{k}, \omega_{n}\right) G_{0}^{-1}\left(\mathbf{k}, \omega_{n}\right)-G_{0}^{-1}\left(\mathbf{k}, \omega_{n}\right) .
$$

The last term can be dropped because we need to keep only the terms describing the transitions involving discrete levels. Also since the transfer matrix does not depend on wave vector $\mathbf{k}$, we may simplify the last expression by taking $\mathbf{k}$ to lie on the Fermi surface, where $\xi_{\mathbf{k}}=0$. Thus we arrive at

$$
\frac{N_{i} J}{N} t\left(\omega_{n}\right)=G_{0, F}^{-1}\left(\omega_{n}\right) G_{m, F}\left(\omega_{n}\right) G_{0, F}^{-1}\left(\omega_{n}\right)
$$

where the subscript $F$ means that the corresponding Green's function has been evaluated at the Fermi surface. We now substitute this expression for the transfer matrix into the term $G_{0}\left(\mathbf{k}, \omega_{n}\right) t\left(\omega_{n}\right) G_{0}\left(\mathbf{k}^{\prime}, \omega_{n}\right) N_{i} J / N$ from Eq. (12) obtaining (after straightforward simplifications) the following result:

$$
\begin{aligned}
& G_{0}\left(\mathbf{k}, \omega_{n}\right) \frac{N_{i} J}{N} t\left(\omega_{n}\right) G_{0}\left(\mathbf{k}^{\prime}, \omega_{n}\right) \\
&=G_{0}\left(\mathbf{k}, \omega_{n}\right) G_{0, F}^{-1}\left(\omega_{n}\right) G_{m, F}\left(\omega_{n}\right) G_{0, F}^{-1}\left(\omega_{n}\right) G_{0}\left(\mathbf{k}^{\prime}, \omega_{n}\right) \\
&=G_{m, F}\left(\omega_{n}\right)+\xi G_{0}\left(\mathbf{k}, \omega_{n}\right) \sigma_{0} \tau_{3} G_{m, F}\left(\omega_{n}\right) \\
& \quad+\xi^{\prime} G_{m, F}\left(\omega_{n}\right) \sigma_{0} \tau_{3} G_{0}\left(\mathbf{k}, \omega_{n}\right) \\
& \quad+\xi \xi^{\prime} G_{0}\left(\mathbf{k}, \omega_{n}\right) \sigma_{0} \tau_{3} G_{m, F}\left(\omega_{n}\right) \sigma_{0} \tau_{3} G_{0}\left(\mathbf{k}^{\prime}, \omega_{n}\right) \\
& \rightarrow \xi \xi^{\prime} G_{0}\left(\mathbf{k}, \omega_{n}\right) \sigma_{0} \tau_{3} G_{m, F}\left(\omega_{n}\right) \sigma_{0} \tau_{3} G_{0}\left(\mathbf{k}^{\prime}, \omega_{n}\right)
\end{aligned}
$$

where $\xi=\xi_{\mathbf{k}}$ and $\xi^{\prime}=\xi_{\mathbf{k}^{\prime}}$, and we keep only the last term because after the substitution back into the self-energy, the first three terms will give zero contribution due to condition (15). Thus, introducing the notation $U_{\mathbf{q} j}(\mathbf{p}-\mathbf{k})=\mathbf{e}_{\mathbf{q} j} \cdot(\mathbf{p}$ $-\mathbf{k}) U(\mathbf{p}-\mathbf{k})$ we rewrite the self-energy in a fully symmetric form

$$
\begin{aligned}
\Sigma_{p h}\left(\mathbf{p}, \omega_{n}\right)= & -T \sum_{n^{\prime}} \sum_{\mathbf{q} j} \sum_{\mathbf{k}, \mathbf{k}^{\prime}} \frac{\hbar D_{\mathbf{q} j}\left(\omega_{n}-\omega_{n^{\prime}}\right)}{2 M N \omega_{\mathbf{q} j}} \\
& \times U_{\mathbf{q} j}(\mathbf{p}-\mathbf{k}) \xi \xi^{\prime} G_{0}\left(\mathbf{k}, \omega_{n^{\prime}}\right) \sigma_{0} \tau_{3} G_{m, F}\left(\omega_{n^{\prime}}\right) \sigma_{0} \tau_{3} \\
& \times G_{0}\left(\mathbf{k}^{\prime}, \omega_{n^{\prime}}\right) U_{\mathbf{q} j}\left(\mathbf{p}-\mathbf{k}^{\prime}\right)
\end{aligned}
$$

It is important to notice that if $\xi \rightarrow \infty$ then $\xi G_{0}\left(\mathbf{k}, \omega_{n}\right) \rightarrow$ $-\sigma_{0} \tau_{3}$ and similarly for $\xi^{\prime} G_{0}\left(\mathbf{k}^{\prime}, \omega_{n}\right)$, resulting in the convergence of the expression for self-energy because of condition (15). The linear terms in $\xi$ and $\xi^{\prime}$ in the expressions for $\xi G_{0}\left(\mathbf{k}, \omega_{n}\right)$ and $\xi^{\prime} G_{0}\left(\mathbf{k}^{\prime}, \omega_{n}\right)$ can be neglected because they give a much smaller contribution associated with the change of their sign crossing the Fermi surface when summing over $\mathbf{k}$ or $\mathbf{k}^{\prime}$. It can also be shown that the dominant contribution to self-energy in Eq. (19) comes from the summation close to the Fermi surface. Thus we may replace $\xi G_{0}\left(\mathbf{k}, \omega_{n^{\prime}}\right)$ with $\sigma_{0} \tau_{3}\left(\omega_{n^{\prime}}^{2}+\Delta^{2}\right) /\left(\omega_{n^{\prime}}^{2}+\xi^{2}+\Delta^{2}\right)$ and integrate the coupling potentials over the Fermi surface. Therefore we have

$$
\begin{aligned}
& \sum_{\mathbf{k}} \frac{\omega_{n^{\prime}}^{2}+\Delta^{2}}{\omega_{n^{\prime}}^{2}+\xi^{2}+\Delta^{2}} U_{\mathbf{q} j}(\mathbf{p}-\mathbf{k}) \\
& \quad=\int_{-\infty}^{\infty} \frac{d \xi}{\omega_{n^{\prime}}^{2}+\xi^{2}+\Delta^{2}} \oint_{S_{F}} \frac{d^{2} \mathbf{k}}{\left|V_{\mathbf{k}}\right|} U_{\mathbf{q} j}(\mathbf{p}-\mathbf{k}) \\
& \quad=\left.\pi N(0) a_{0}^{3} \sqrt{\omega_{n^{\prime}}^{2}+\Delta^{2}} \int \frac{d O_{\mathbf{k}}}{4 \pi} U_{\mathbf{q} j}(\mathbf{p}-\mathbf{k})\right|_{k=k_{F}},
\end{aligned}
$$

where $S_{F}$ is the Fermi surface, $N(0)$ is the density of states at the Fermi level in the normal state per spin, $a_{0}^{3}$ is the volume of the elementary cell, and $k_{F}$ is the Fermi momentum. Using this result and introducing the notation

$$
\tilde{U}_{\mathbf{q} j}(\mathbf{p})=\left.N(0) a_{0}^{3} \int \frac{d O_{\mathbf{k}}}{4 \pi} U_{\mathbf{q} j}(\mathbf{p}-\mathbf{k})\right|_{k=k_{F}},
$$

we arrive at the following expression for self-energy:

$$
\begin{aligned}
\Sigma_{p h}\left(\mathbf{p}, \omega_{n}\right)= & -T \pi^{2} \sum_{n^{\prime}} \sum_{\mathbf{q} j} \frac{\hbar D_{\mathbf{q} j}\left(\omega_{n}-\omega_{n^{\prime}}\right)}{2 M N \omega_{\mathbf{q} j}} \\
& \times \widetilde{U}_{\mathbf{q} j}(\mathbf{p}) G_{m, F}\left(\omega_{n^{\prime}}\right) \tilde{U}_{\mathbf{q} j}(\mathbf{p})\left(\omega_{n^{\prime}}^{2}+\Delta^{2}\right) .
\end{aligned}
$$

For comparison, the self-energy due to the conventional electron-phonon interaction in a superconductor can be written as

$$
\begin{aligned}
\Sigma_{e-p h}\left(\mathbf{p}, \omega_{n}\right)= & -T \sum_{n^{\prime}} \sum_{\mathbf{p}^{\prime} j} \frac{\hbar D_{\left(\mathbf{p}-\mathbf{p}^{\prime}\right) j}\left(\omega_{n}-\omega_{n^{\prime}}\right)}{2 M N \omega_{\left(\mathbf{p}-\mathbf{p}^{\prime}\right) j}} \\
& \times\left[\mathbf{e}_{\left(\mathbf{p}-\mathbf{p}^{\prime}\right) j} \cdot\left(\mathbf{p}-\mathbf{p}^{\prime}\right) u_{e i}\left(\mathbf{p}-\mathbf{p}^{\prime}\right)\right] G\left(\mathbf{p}^{\prime}, \omega_{n^{\prime}}\right),
\end{aligned}
$$

where $u_{e i}$ is the electron-ion potential. In this notation the structure of the self-energy for the two interaction mechanisms, with phonon exchange between continuum and discrete states and all states within the continuum, looks very similar. Expression (22) needs minor renormalization. If $\omega_{n^{\prime}} \rightarrow \infty$ the terms under the sum become constant, leading to divergence of the real part of the self-energy. This divergence originated because we integrated over $d \xi$ and $d \xi^{\prime}$ with the coupling potentials already averaged over the Fermi surface. In original expression (19) it is absent because of condition (15). We may eliminate this artificial singularity by taking away the limiting value of self-energy when $\omega_{n^{\prime}} \rightarrow \infty$. How- 
ever it is only important for the real part of the self-energy, which for the dilute limit is of no interest.

Following the standard procedure and using spectral representations for the electron and phonon Green's functions, we sum over Matsubara frequencies. Performing an analytic continuation from the imaginary to the real axes using the substitution $i \omega_{n} \rightarrow \omega+i \delta$, we obtain for the imaginary part of the self-energy,

$$
\begin{aligned}
\operatorname{Im} \Sigma_{p h}= & -\frac{\pi \Delta}{4} \sum_{\mathbf{q} j} \frac{\hbar}{2 M N \omega_{\mathbf{q} j}} \int_{-\infty}^{\infty} d z \int_{-\Delta}^{\Delta} d z^{\prime} \\
& \times \operatorname{Im} D_{\mathbf{q} j}(z) \widetilde{U}_{\mathbf{q} j}(\mathbf{p}) \operatorname{Im} G_{m}\left(z^{\prime}\right) \widetilde{U}_{\mathbf{q} j}(\mathbf{p}) \\
& \times\left(\tanh \frac{z^{\prime}}{2 T}+\operatorname{coth} \frac{z}{2 T}\right) \delta\left(\epsilon-z-z^{\prime}\right)
\end{aligned}
$$

where we have introduced

$$
G_{m}\left(z^{\prime}\right)=G_{m, F}\left(z^{\prime}\right)\left(\Delta^{2}-z^{\prime 2}\right) / \Delta=\left(\begin{array}{cc}
G\left(z^{\prime}\right) \sigma_{0} & F\left(z^{\prime}\right) i \sigma_{2} \\
-F^{+}\left(z^{\prime}\right) i \sigma_{2} & \widetilde{G}\left(z^{\prime}\right) \sigma_{0}
\end{array}\right) .
$$

Here $G\left(z^{\prime}\right), \widetilde{G}\left(z^{\prime}\right), F\left(z^{\prime}\right)$, and $F^{+}\left(z^{\prime}\right)$ are the spatially averaged electronic Green's functions obtained within the model of Müller-Hartmann and Zittartz in the dilute limit. The limits of integration in the second integral were set between $-\Delta$ and $\Delta$ to emphasize that we are interested in the contribution to self-energy arising from inelastic transitions involving continuum and discrete states.

Calculation of the product of three matrices $\tilde{U}_{\mathbf{q} j}(\mathbf{p}) \operatorname{Im} G_{m}\left(z^{\prime}\right) \tilde{U}_{\mathbf{q} j}(\mathbf{p})$ is straightforward leading to

$$
\begin{aligned}
& \tilde{U}_{\mathbf{q} j}(\mathbf{p}) \operatorname{Im} G_{m}\left(z^{\prime}\right) \tilde{U}_{\mathbf{q} j}(\mathbf{p}) \\
& \quad=\left(\begin{array}{cc}
\operatorname{Im} G\left(z^{\prime}\right)|\tilde{U}|^{2} \sigma_{0} & -\operatorname{Im} F\left(z^{\prime}\right) \tilde{U} i \sigma_{2} \tilde{U}^{t^{*}} \\
\operatorname{Im} F\left(z^{\prime}\right) U^{t} i \sigma_{2} \tilde{U}^{*} & \operatorname{Im} G\left(z^{\prime}\right)\left|\tilde{U}^{t}\right|^{2} \sigma_{0}
\end{array}\right),
\end{aligned}
$$

where we introduced

$$
\tilde{U}_{\mathbf{q} j}(\mathbf{p})=\left(\begin{array}{cc}
\tilde{U} & 0 \\
0 & -\tilde{U}^{t}
\end{array}\right)
$$

with $\tilde{U}^{t}$ as the transposed matrix. After statistical averaging of spin variables we arrive at

$$
\begin{aligned}
|\tilde{U}|^{2} \sigma_{0}= & {\left[\left\langle\left\langle\mathbf{e}_{\mathbf{q} j} \cdot(\mathbf{p}-\mathbf{k}) u_{1}(\mathbf{p}-\mathbf{k})\right\rangle\right\rangle^{2}\right.} \\
& \left.+S(S+1)\left\langle\left\langle\mathbf{e}_{\mathbf{q} j} \cdot(\mathbf{p}-\mathbf{k}) u_{2}(\mathbf{p}-\mathbf{k})\right\rangle\right\rangle^{2}\right] \sigma_{0}, \\
\tilde{U} i \sigma_{2} \tilde{U}^{t *}= & {\left[\left\langle\left\langle\mathbf{e}_{\mathbf{q} j} \cdot(\mathbf{p}-\mathbf{k}) u_{1}(\mathbf{p}-\mathbf{k})\right\rangle\right\rangle^{2}\right.} \\
& \left.-S(S+1)\left\langle\left\langle\mathbf{e}_{\mathbf{q} j} \cdot(\mathbf{p}-\mathbf{k}) u_{2}(\mathbf{p}-\mathbf{k})\right\rangle\right\rangle^{2}\right] i \sigma_{2},
\end{aligned}
$$

where the $\langle\langle\ldots\rangle\rangle$ denotes the average over the Fermi surface according to

$$
\begin{aligned}
& \left\langle\left\langle\mathbf{e}_{\mathbf{q} j} \cdot(\mathbf{p}-\mathbf{k}) u_{1,2}(\mathbf{p}-\mathbf{k})\right\rangle\right\rangle \\
& \quad=\left.N(0) a_{0}^{3} \int \frac{d O_{\mathbf{k}}}{4 \pi} \mathbf{e}_{\mathbf{q} j} \cdot(\mathbf{p}-\mathbf{k}) u_{1,2}(\mathbf{p}-\mathbf{k})\right|_{k=k_{F}} .
\end{aligned}
$$

Introducing coupling constants through

$$
\begin{gathered}
\left|g_{\mathbf{q} j}^{ \pm}(\mathbf{p})\right|^{2}=\left|g_{\mathbf{q} j}^{(1)}(\mathbf{p})\right|^{2} \pm S(S+1)\left|g_{\mathbf{q} j}^{(2)}(\mathbf{p})\right|^{2}, \\
\left|g_{\mathbf{q} j}^{(1,2)}(\mathbf{p})\right|=\sqrt{\frac{\hbar}{2 M N \omega_{\mathbf{q} j}}}\left\langle\left\langle\mathbf{e}_{\mathbf{q} j} \cdot(\mathbf{p}-\mathbf{k}) u_{1,2}(\mathbf{p}-\mathbf{k})\right\rangle\right\rangle,
\end{gathered}
$$

we define the analog of the Eliashberg function for electronphonon interaction with transitions between continuum and discrete states

$$
\alpha_{1,2}^{2}(z) \Phi(z)=\frac{\pi}{2} \Delta \sum_{\mathbf{q} j} \delta\left(z-\omega_{\mathbf{q} j}\right)\left\langle\left|g_{\mathbf{q} j}^{1,2}(\mathbf{p})\right|^{2}\right\rangle,
$$

where $\Phi(\Omega)$ is the phonon density of states and $\langle\ldots\rangle$ is the symbol for averaging over directions of vector $\mathbf{p}$. For comparison the conventional Eliashberg function is given by the standard expression which can be written as

$$
\alpha^{2}(z) \Phi(z)=\frac{1}{\hbar v_{F}} \sum_{\mathbf{q}, j} \delta\left(z-\omega_{\mathbf{q}, j}\right)\left|g_{j}(\mathbf{q})\right|^{2}\left\langle\delta\left(|\mathbf{p}-\mathbf{q}|-p_{F}\right)\right\rangle,
$$

where $g_{j}(\mathbf{q})=\sqrt{\hbar / 2 M N \omega_{\mathbf{q}, j}} \mathbf{e}_{\mathbf{q}, j} \cdot \mathbf{q} u_{e i}(\mathbf{q})$. Using Eq. (31) we may write the expression for the imaginary part of selfenergy in the final form

$$
\begin{aligned}
\operatorname{Im} \Sigma_{p h}(p, \omega)= & \left(\begin{array}{cc}
\Sigma_{1, p h} \sigma_{0} & \Sigma_{2, p h} i \sigma_{2} \\
-\Sigma_{2, p h}^{+} i \sigma_{2} & \widetilde{\Sigma}_{1, p h} \sigma_{0}
\end{array}\right)=-\pi \int_{-\infty}^{\infty} d z \int_{-\Delta}^{\Delta} d z^{\prime}\left[\left(\tanh \frac{z^{\prime}}{2 T}+\operatorname{coth} \frac{z}{2 T}\right) \delta\left(\omega-z-z^{\prime}\right)-\left(\tanh \frac{z^{\prime}}{2 T}-\operatorname{coth} \frac{z}{2 T}\right)\right. \\
& \left.\times \delta\left(\omega+z-z^{\prime}\right)\right] \Phi(z)\left(\begin{array}{ll}
\operatorname{Im} G\left(z^{\prime}\right)\left[\alpha_{1}^{2}(z)+S(S+1) \alpha_{2}^{2}(z)\right] \sigma_{0} & -\operatorname{Im} F\left(z^{\prime}\right)\left[\alpha_{1}^{2}(z)-S(S+1) \alpha_{2}^{2}(z)\right] i \sigma_{2} \\
\operatorname{Im} F\left(z^{\prime}\right)\left[\alpha_{1}^{2}(z)-S(S+1) \alpha_{2}^{2}(z)\right] i \sigma_{2} & \operatorname{Im} G\left(z^{\prime}\right)\left[\alpha_{1}^{2}(z)+S(S+1) \alpha_{2}^{2}(z)\right] \sigma_{0}
\end{array}\right) .
\end{aligned}
$$


Deriving this expression we substitute $\operatorname{Im} D_{\mathbf{q} j}(z)=2 \pi[\delta(z$ $\left.\left.-\omega_{\mathbf{q} j}\right)-\delta\left(z+\omega_{\mathbf{q} j}\right)\right]$ and then transform the result reversing the sign of $z$ in the second term.

\section{TRANSITION RATES}

\section{A. General expression for transition rates}

In order to analyze the rates of QP transitions from an initial state $(\mathbf{p}, \epsilon)$ belonging to a continuum, we calculate the Green's function including self-energy (33). This calculation can be carried out within the Eliashberg model. In the dilute limit we take the renormalization parameter as being fully determined through interactions only within the continuum, thus, neglecting all effects due to impurities. Solving the equation for the one-particle Green's function,

$$
G^{-1}(\mathbf{p}, \epsilon)=G_{0}^{-1}(\mathbf{p}, \epsilon)-\Sigma_{p h}(\mathbf{p}, \epsilon),
$$

we identify the poles describing inelastic interactions involving the discrete states. Taking $\epsilon=\epsilon^{\prime}-i \Gamma$ and separating the real and imaginary parts, we obtain the expression for transition rates in the form

$$
\begin{aligned}
\Gamma p(\epsilon)= & -\frac{1}{2 \epsilon Z_{1}(0)}\left[\epsilon \operatorname{Im}\left(\Sigma_{1, p h}+\widetilde{\Sigma}_{1, p h}\right)-\xi_{p}\left(\Sigma_{1, p h}-\widetilde{\Sigma}_{1, p h}\right)\right. \\
& \left.+\Delta \operatorname{Im}\left(\Sigma_{2, p h}+\Sigma_{2, p h}^{+}\right)\right]=-\frac{1}{Z_{1}(0)}\left[\Sigma_{1, p h}+\frac{\Delta}{\epsilon} \Sigma_{2, p h}\right],
\end{aligned}
$$

where $Z_{1}(0)$ is the real part of the renormalization parameter. The last relation holds true because from Eq. (33) it follows that $\tilde{\Sigma}_{1, p h}=\Sigma_{1, p h}$ and $\Sigma_{2, p h}^{+}=\Sigma_{2, p h}$.

Using the expression for self-energy (33) and substituting it into Eq. (35), we obtain

$$
\begin{aligned}
\Gamma(\epsilon)= & \frac{\pi}{Z_{1}(0)} \int_{-\infty}^{\infty} d z \int_{-\Delta}^{\Delta} d z^{\prime} \Phi(z)\left\{\alpha_{1}^{2}(z) \operatorname{Im}\left[G\left(z^{\prime}\right)-\frac{\Delta}{\epsilon} F\left(z^{\prime}\right)\right]\right. \\
& \left.+S(S+1) \alpha_{2}^{2}(z) \operatorname{Im}\left[G\left(z^{\prime}\right)+\frac{\Delta}{\epsilon} F\left(z^{\prime}\right)\right]\right\}\left\{\tanh \left(\frac{z^{\prime}}{2 T}\right)\right. \\
& \left.+\operatorname{coth}\left(\frac{z}{2 T}\right)\right] \delta\left(\epsilon-z-z^{\prime}\right)-\left[\tanh \left(\frac{z^{\prime}}{2 T}\right)\right. \\
& \left.\left.-\operatorname{coth}\left(\frac{z}{2 T}\right)\right] \delta\left(\epsilon+z-z^{\prime}\right)\right\} .
\end{aligned}
$$

It is important to note that the sign of the contribution of the anomalous Green's function, $F$, in the combination of the Green's functions in Eq. (36), which defines the coherence factors for various interactions, is different for deformationpotential and spin-lattice couplings. This is because of the reversal of sign associations for interactions involving spins. ${ }^{14}$ The exact expressions for $\operatorname{Im} G(\epsilon)$ and $\operatorname{Im} F(\epsilon)$ are obtained using Eq. (25) and the expression for the Green's function derived by Müller-Hartmann and Zittartz. They have the form
$G(\epsilon)=\frac{\Delta^{2}-\epsilon^{2}}{\Delta} \frac{\tilde{\epsilon}(\epsilon)}{\tilde{\epsilon}^{2}(\epsilon)-\widetilde{\Delta}^{2}(\epsilon)} ; \quad F(\epsilon)=\frac{\Delta^{2}-\epsilon^{2}}{\Delta} \frac{\tilde{\Delta}(\epsilon)}{\tilde{\epsilon}^{2}(\epsilon)-\widetilde{\Delta}^{2}(\epsilon)}$.

These functions are closely related to the quasiclassical Green's functions for a homogeneous case,

$$
G_{\mathrm{MHZ}}(\epsilon)=\frac{\widetilde{\epsilon}(\epsilon)}{\sqrt{\widetilde{\epsilon}^{2}(\epsilon)-\widetilde{\Delta}^{2}(\epsilon)}} ; \quad F_{\mathrm{MHZ}}(\epsilon)=\frac{\tilde{\Delta}(\epsilon)}{\sqrt{\widetilde{\epsilon}^{2}(\epsilon)-\widetilde{\Delta}^{2}(\epsilon)}} .
$$

The imaginary parts of $G(\epsilon)$ and $F(\epsilon)$ can be expressed in terms of real parts of $G_{\mathrm{MHZ}}(\epsilon)$ and $F_{\mathrm{MHZ}}(\epsilon)$. The energy $\tilde{\epsilon}$ and the order parameter $\tilde{\Delta}$ in Eqs. (37) and (38) satisfy the following equations: ${ }^{7}$

$$
\tilde{\boldsymbol{\epsilon}}=\boldsymbol{\epsilon}+\Delta \Sigma_{1}(\widetilde{\boldsymbol{\epsilon}}, \widetilde{\Delta}) ; \quad \widetilde{\Delta}=\Delta+\Delta \Sigma_{2}(\widetilde{\boldsymbol{\epsilon}}, \widetilde{\Delta}),
$$

where

$$
\begin{aligned}
& \Sigma_{1}(y, \Delta)=-\frac{c}{i \pi} \frac{\sqrt{y^{2}-1}}{y^{2}-y_{0}^{2}} y\left(1-y_{0}\right), \\
& \Sigma_{2}(y, \Delta)=\frac{c}{i \pi} \frac{\sqrt{y^{2}-1}}{y^{2}-y_{0}^{2}} y_{0}\left(1-y_{0}\right)
\end{aligned}
$$

are the elements of the self-energy matrix for scattering by Kondo impurities. Here $y=\epsilon / \Delta, y_{0}=\epsilon_{0} / \Delta<1$, and $\epsilon_{0}$ is the discrete intragap level. To find solutions to the main terms, we may use the simplified equations obtained from Eq. (39) by taking the renormalized parameters $\tilde{y}=\tilde{\epsilon} / \tilde{\Delta}$ and $\tilde{y}_{0}$ $=\epsilon_{0} / \widetilde{\Delta}$ in the denominators in Eq. (40). The simplified equations then become

$$
\begin{gathered}
\tilde{y}=y-\bar{c} \frac{y}{|y|}\left(\frac{1}{\tilde{y}-\tilde{y}_{0}}-\frac{1}{\tilde{y}+\tilde{y}_{0}}\right), \\
\widetilde{\Delta}=\Delta+\bar{c} \frac{y}{|y|} \Delta\left(\frac{1}{\tilde{y}-\tilde{y}_{0}}-\frac{1}{\tilde{y}+\tilde{y}_{0}}\right),
\end{gathered}
$$

where $\bar{c}=c \frac{\sqrt{1-y^{2}}|y|\left(1-y_{0}\right)}{2 \pi y_{0}}$. In order to solve the simplified equations we first note that from Eq. (40) $\Sigma_{2}=-y_{0} / y \Sigma_{1}$. Above the gap edge both $\left|\Sigma_{1}\right| \sim c$ and $\left|\Sigma_{2}\right| \sim c$; as pointed out above we ignore these corrections. Inside the gap the solution for the imaginary part $\Sigma_{1}^{\prime \prime}$ has the form

$$
\Sigma_{1}^{\prime \prime}=\sqrt{\bar{c}-\frac{1}{4}\left(|y|-y_{0}\right)^{2}} \Theta\left[\bar{c}-\frac{1}{4}\left(|y|-y_{0}\right)^{2}\right] .
$$

Within the range $\Sigma_{1}^{\prime \prime} \neq 0$, the real part of the self-energy $\Sigma_{1}^{\prime}$ is given by

$$
\Sigma_{1}^{\prime}=\frac{1}{2}\left(y_{0}-|y|\right)+\frac{\bar{c}}{4 y_{0}} .
$$

Outside this range, but still inside the gap, $\Sigma_{1}^{\prime}$ remains finite with a dependence on impurity concentration changing from $\sqrt{c}$ at the edge of the $\Sigma_{1}^{\prime \prime} \neq 0$ range to $c$ away from it. 


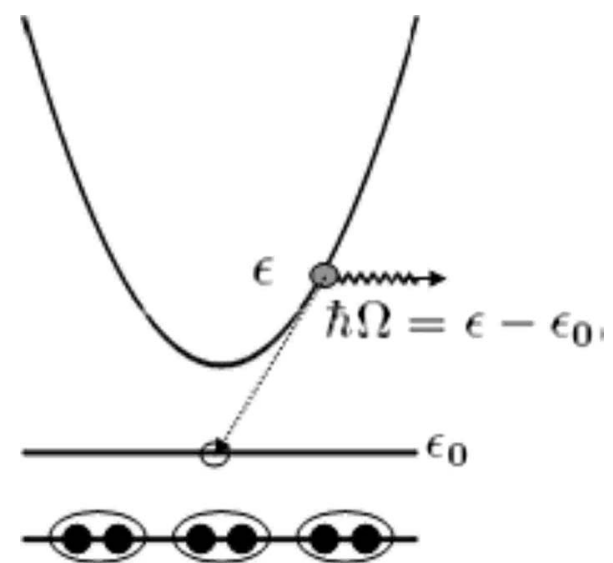

FIG. 2. Quasiparticle trapping by magnetic impurity. A quasiparticle initially in the continuum spectrum at energy $\epsilon$ undergoes an inelastic transition to a discrete level $\epsilon_{0}$ with emission of a phonon $\hbar \Omega=\epsilon-\epsilon_{0}$.

Finally, inside the gap we obtain

$$
\begin{aligned}
\operatorname{Im} G(y)= & \operatorname{Im} F(y)=-\frac{\left(1+y_{0}\right)^{5 / 2}}{1-y_{0}} \sqrt{\bar{c}-\frac{1}{4}\left(|y|-y_{0}\right)^{2} \Theta} \\
& \times\left[\bar{c}-\frac{1}{4}\left(|y|-y_{0}\right)^{2}\right], \\
& \operatorname{Re} G_{\mathrm{MHZ}}(y)=-\frac{1-y_{0}}{\left(1-y_{0}^{2}\right)^{3 / 2}} \operatorname{Im} G(y) .
\end{aligned}
$$

These expressions describe the normalized density of bound states inside the gap in a superconductor with magnetic impurities. This distribution is sharp, with both its width and height being proportional to $\sqrt{c}$. It is easy to confirm that $\int_{-1}^{1} d y \operatorname{Re} G_{\mathrm{MHZ}}(y)=c$, corresponding to one QP bound state for each impurity atom.

Finally, the classical spin limit corresponds to $S \rightarrow \infty$, while simultaneously $J \rightarrow 0$ so that $J S=$ constant. In this limit the localized spin acts as a local magnetic field. We note that the expressions for the transfer matrix $t\left(\omega_{n}\right)$, and hence, for the self-energies $\Sigma_{1}$ and $\Sigma_{2}$ [Eq. (40)] are identical to those for a classical impurity spin, ${ }^{3}$ except that the position $y_{0}$ of the discrete level is different. Therefore all our results will also correctly describe transitions from continuum to bound states associated with classical impurity spins.

\section{B. Quasiparticle transition from continuum to bound state: Trapping}

Using the Green's functions given by Eqs. (44) and (35) we may now analyze different inelastic transitions. First, a QP initially in the continuum state may become trapped on a state bound to an impurity. This process is schematically illustrated in Fig. 2. For the trapping rates we obtain

$$
\begin{aligned}
\frac{1}{\tau_{\text {trap }}(\epsilon)}= & \frac{2 \Gamma(\omega)}{\hbar}=\int_{0}^{\Delta} \frac{d \epsilon^{\prime}\left(\epsilon-\epsilon^{\prime}\right)}{\Delta^{2}}\left[\operatorname{Im} G\left(\epsilon^{\prime}\right)\left(\frac{1}{\tau_{1}}+\frac{1}{\tau_{2}}\right)\right. \\
& \left.-\frac{\Delta}{\epsilon} \operatorname{Im} F\left(\epsilon^{\prime}\right)\left(\frac{1}{\tau_{1}}-\frac{1}{\tau_{2}}\right)\right]\left[n\left(\epsilon-\epsilon^{\prime}\right)+1\right]\left[1-f\left(\epsilon^{\prime}\right)\right] .
\end{aligned}
$$

The integrand in this expression contains a product of sharp and smooth functions. For a narrow (sharp) impurity band inside the gap, replacing the smooth functions by their values at the location of the discrete level, we obtain

$$
\begin{gathered}
\frac{1}{\tau_{\text {trap }}(\epsilon)}=\frac{1}{\tau_{\text {trap }, 1}(\epsilon)}+\frac{1}{\tau_{\text {trap }, 2}(\epsilon)}, \\
\frac{1}{\tau_{\text {trap }, 1}(\epsilon)}=c \frac{1}{\tau_{1}}\left(1-\frac{\epsilon_{0}}{\epsilon}\right)\left(\frac{\epsilon}{\Delta}-1\right)\left[n\left(\epsilon-\epsilon_{0}\right)+1\right]\left[1-f\left(\epsilon_{0}\right)\right], \\
\frac{1}{\tau_{\text {trap }, 2}(\epsilon)}=c \frac{1}{\tau_{2}}\left(1-\frac{\epsilon_{0}}{\epsilon}\right)\left(\frac{\epsilon}{\Delta}+1\right)\left[n\left(\epsilon-\epsilon_{0}\right)+1\right]\left[1-f\left(\epsilon_{0}\right)\right] .
\end{gathered}
$$

Here $n(\epsilon)$ and $f(\epsilon)$ are the phonon and QP distribution functions, and the notations for the trapping times are $\tau_{\text {trap, } 1}$ for deformation-potential interaction and $\tau_{\text {trap,2 }}$ for the spinlattice interaction. The characteristic relaxation times for phonon-assisted scattering on a magnetic impurity in the host lattice $\tau_{(1,2)}$ can be written in the form

$$
\begin{aligned}
\frac{1}{\tau_{1}}= & \frac{1}{\tau_{0}} \frac{\alpha_{1}^{2}(\Delta)}{\alpha^{2}(\Delta)}\left(\frac{\Delta}{T_{c}}\right)^{3} \frac{\left(1-y_{0}^{2}\right)^{3 / 2}}{1-y_{0}} ; \\
& \frac{1}{\tau_{2}}=\frac{S(S+1)}{\tau_{0}} \frac{\alpha_{2}^{2}(\Delta)}{\alpha^{2}(\Delta)}\left(\frac{\Delta}{T_{c}}\right)^{3} \frac{\left(1-y_{0}^{2}\right)^{3 / 2}}{1-y_{0}},
\end{aligned}
$$

where $\alpha$ is the parameter entering Eliashberg constant (32), $\tau_{0}$ is the superconductor characteristic relaxation time for deformation-potential coupling, and $T_{c}$ is the critical temperature. The characteristic times describing inelastic transitions between continuum and discrete states for both deformation-potential $\left(\tau_{1}\right)$ and spin-lattice interactions $\left(\tau_{2}\right)$ depend on properties of both the host lattice and magnetic impurity.

For QPs at the edge of the gap, $\epsilon=\Delta$, and the interaction via the deformation potential vanish so that $\tau_{\text {trap }, 1}=\infty$, and trapping is due only to the spin-lattice interaction. This occurs because of condition (44) for the discrete state, resulting in nullification of the coherence factor for deformationpotential coupling. In contrast, spin-lattice interaction, because of the sign reversal in the second term of the coherence factor, dominates the trapping rate for all energies close to the edge. This raises the possibility of measuring the characteristic spin-lattice relaxation time $\tau_{2}$ directly in experiments where nonequilibrium QPs are excited to the states close to the edge of the gap. 


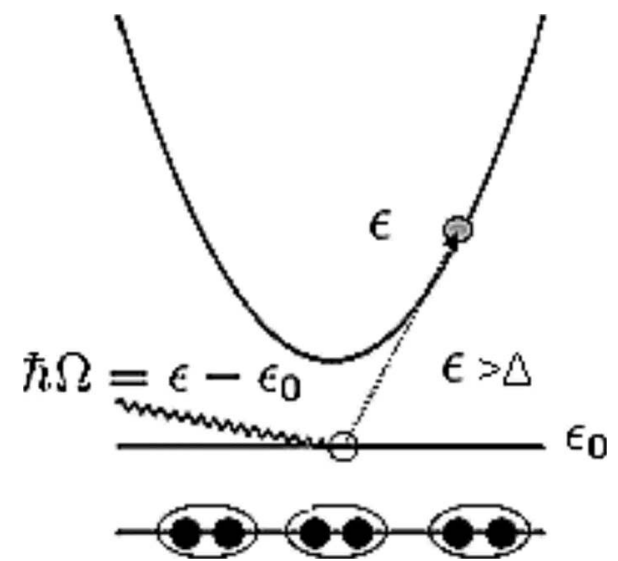

FIG. 3. Activation of a quasiparticle from the bound state at magnetic impurity by a phonon $\hbar \Omega=\epsilon-\epsilon_{0}$.

\section{Activation of bound quasiparticle to continuum state: Detrapping}

Detrapping of a quasiparticle, which is bound to a magnetic impurity, occurs when it absorbs thermal phonons with sufficient energy to excite it into the continuum spectrum. This process is illustrated in Fig. 3.

The rate of detrapping from the localized state can be calculated without direct evaluation of the broadening of the bound state due to interaction with phonons. This is because we are not interested in transitions from discrete states to an individual state inside the continuum but only in the activation rate due to transitions into all available states. Therefore we obtain an activation rate by balancing scattering-in and scattering-out rates for the bound state at thermal equilibrium. The result is

$$
\frac{1}{\tau_{\text {detrap }}}=\frac{1}{c} \int_{\Delta}^{\infty} \frac{d \epsilon}{\Delta} \rho(\epsilon) \frac{f_{0}(\epsilon)}{f_{0}\left(\epsilon_{0}\right)} \frac{1}{\tau_{\text {trap }}(\epsilon)},
$$

where $f_{0}(\epsilon)$ is the Fermi distribution function,

$$
\begin{aligned}
\frac{1}{\tau_{\text {detrap }}}= & \frac{1}{\sqrt{2}} \int_{1-\epsilon_{0} / \Delta}^{\infty} d z \frac{z \exp \left(-z \frac{\Delta}{T}\right)}{\sqrt{z+\frac{\epsilon_{0}}{\Delta}-1}}\left[\frac{1}{\tau_{1}}\left(z+\frac{\epsilon_{0}}{\Delta}-1\right)+\frac{2}{\tau_{2}}\right] \\
\simeq & \frac{\sqrt{\pi}}{4}\left(\frac{T}{\Delta}\right)^{3 / 2} \exp \left(-\frac{\Delta-\epsilon_{0}}{T}\right)\left[\frac{1}{\tau_{1}}\left(3+2 \frac{\Delta-\epsilon_{0}}{T}\right) \frac{T}{\Delta}\right. \\
& \left.+\frac{4}{\tau_{2}}\left(1+\frac{\Delta-\epsilon_{0}}{T}\right)\right] .
\end{aligned}
$$

At low temperatures most final states for activated QPs are close to the gap edge. For these transitions as well as for trapping of QPs, which are initially close to the gap edge, the dominant interactions are through spin-lattice interaction.

\section{Interaction of quasiparticle in continuum with quasiparticle in bound state: Recombination}

The recombination rate via a bound state calculated from Eqs. (36) and (44) is given by

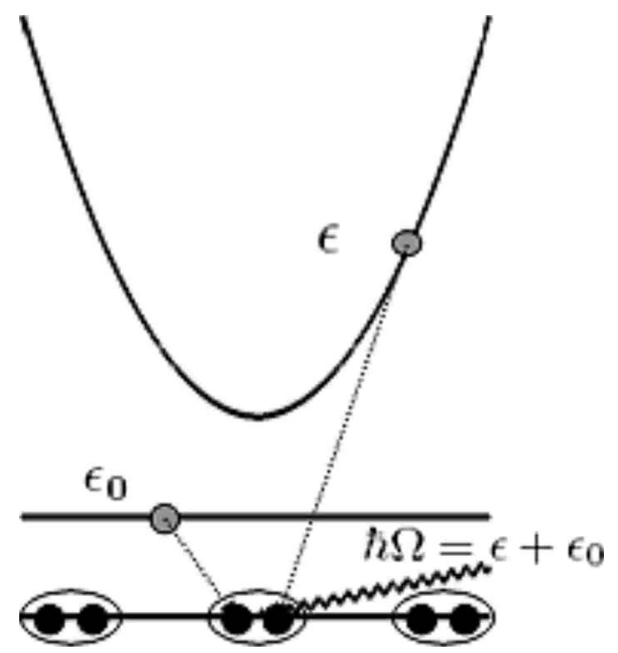

FIG. 4. A quasiparticle in a continuum state at $\epsilon$ recombines with a quasiparticle bound at a magnetic impurity at $\epsilon_{0}$, emitting a phonon with energy $\hbar \Omega=\epsilon+\epsilon_{0}$.

$$
\begin{aligned}
\Gamma_{R, t}(\epsilon)= & \int_{0}^{\Delta} \frac{d \epsilon^{\prime}\left(\epsilon+\epsilon^{\prime}\right)}{\Delta^{2}}\left[\operatorname{Im} G\left(\epsilon^{\prime}\right)\left(\frac{1}{\tau_{1}}+\frac{1}{\tau_{2}}\right)\right. \\
& \left.+\frac{\Delta}{\epsilon} \operatorname{Im} F\left(\epsilon^{\prime}\right)\left(\frac{1}{\tau_{1}}-\frac{1}{\tau_{2}}\right)\right]\left[n\left(\epsilon+\epsilon^{\prime}\right)+1\right] f\left(\epsilon^{\prime}\right) \\
= & c \frac{\epsilon+\epsilon_{0}}{\Delta}\left[\frac{1}{\tau_{1}}\left(1+\frac{\Delta}{\epsilon}\right)+\frac{1}{\tau_{2}}\left(1-\frac{\Delta}{\epsilon}\right)\right] \\
& \times\left[n\left(\epsilon+\epsilon_{0}\right)+1\right] f\left(\epsilon_{0}\right) .
\end{aligned}
$$

Schematically this process is shown in Fig. 4.

The expression can be written in a more familiar form by introducing the appropriate recombination coefficient $R_{t}$ and density of trapped QPs $n_{t}$,

$$
\begin{aligned}
& \Gamma_{R, t}(\epsilon)=R_{t} n_{t} ; \\
& R_{t}=\frac{1}{2 N(0) \Delta} \frac{\epsilon+\epsilon_{0}}{\Delta}\left[\frac{1}{\tau_{1}}\left(1+\frac{\Delta}{\epsilon}\right)+\frac{1}{\tau_{2}}\left(1-\frac{\Delta}{\epsilon}\right)\right],
\end{aligned}
$$

which describes the maximum recombination rate in the absence of a phonon bottleneck effect.

For recombination of a QP at the edge of the gap $(\epsilon=\Delta)$ with another QP, which is bound to an impurity, the coherence factor vanishes for spin-lattice coupling but remains finite for deformation-potential coupling. Therefore by measuring the recombination rate at low temperatures for a nonequilibrium QP distribution localized at the gap edge, we may directly determine the characteristic relaxation time $\tau_{1}$.

Figures 5-7 summarize our discussion of QP trapping and on-trap recombination, and show the dependences of normalized trapping rate (in units of $\tau_{0} / c$ ) and on-trap recombination coefficient (in units of standard recombination coefficient $R$ ) on various parameters for quasiparticles with the initial state at the edge of the gap. 


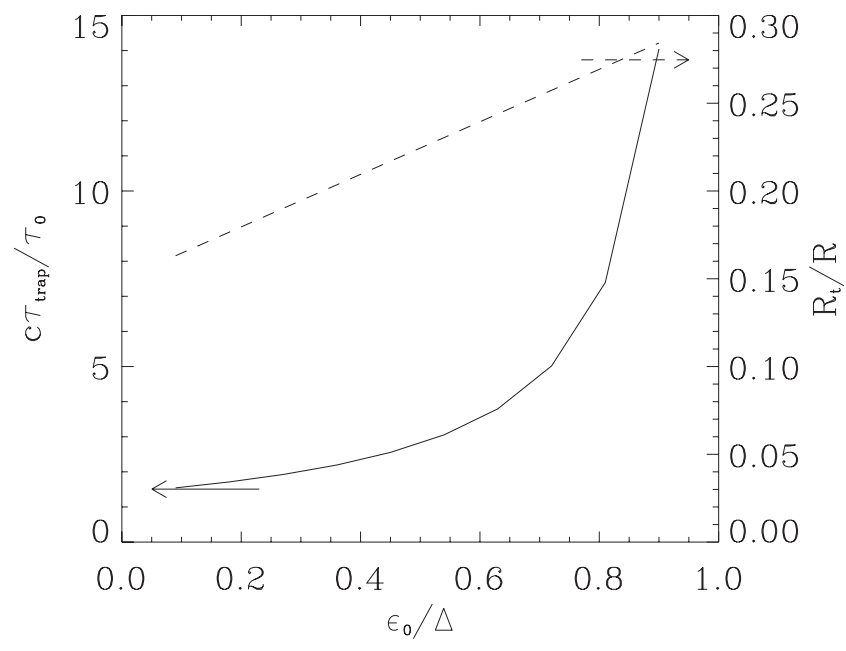

FIG. 5. Normalized trapping time and on-trap recombination coefficient as functions of the position of the impurity level.

\section{E. Breaking of Cooper pairs by phonons below $2 \Delta$ threshold}

In a superconductor with discrete intragap states Cooper pairs can be broken by a phonon with energy below $2 \Delta$ as shown in Fig. 8. The only requirement is that a phonon has a sufficiently high energy to activate one of the correlated electrons from the Fermi level to a bound state while releasing the second electron into a continuum of states above the gap edge. Thus the phonon energy must satisfy the condition $\hbar \Omega \geq \Delta+\epsilon_{0}$. To calculate phonon-scattering rates in a superconductor with Kondo impurities, we must consider possible transitions to bound states. These are taken into account by the extra contribution to phonon self-energy. In the corresponding Feynman diagram, shown in Fig. 9, this is given by one of the lines being the Green's function describing continuum states, while the other representing the discrete intragap states. Repeating the same arguments as in the derivation of the electron self-energy, we derive the following expression corresponding to Fig. 9:

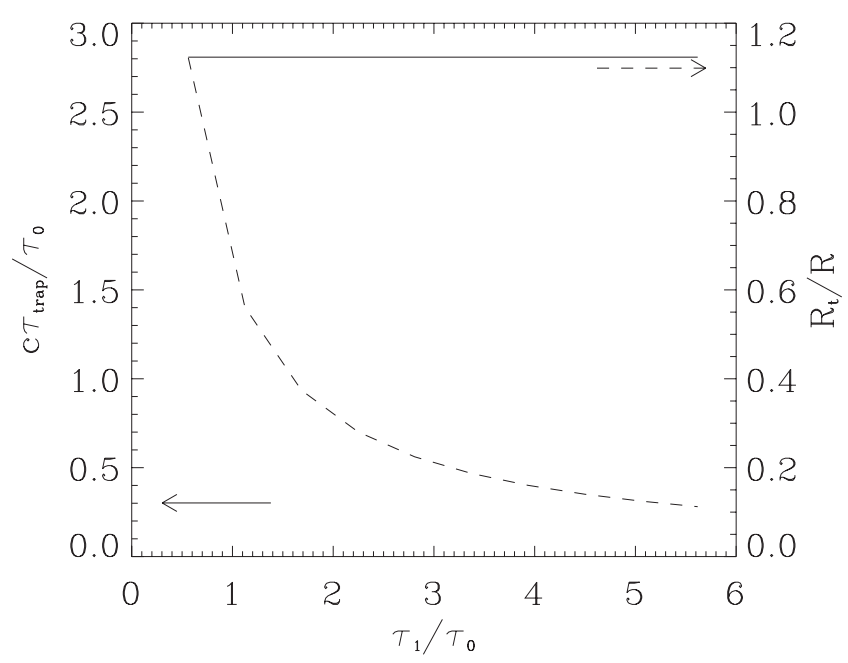

FIG. 6. Normalized trapping time and on-trap recombination coefficient as functions of characteristic time $\tau_{1}$ for coupling of the defect level to phonons due to deformation potential.

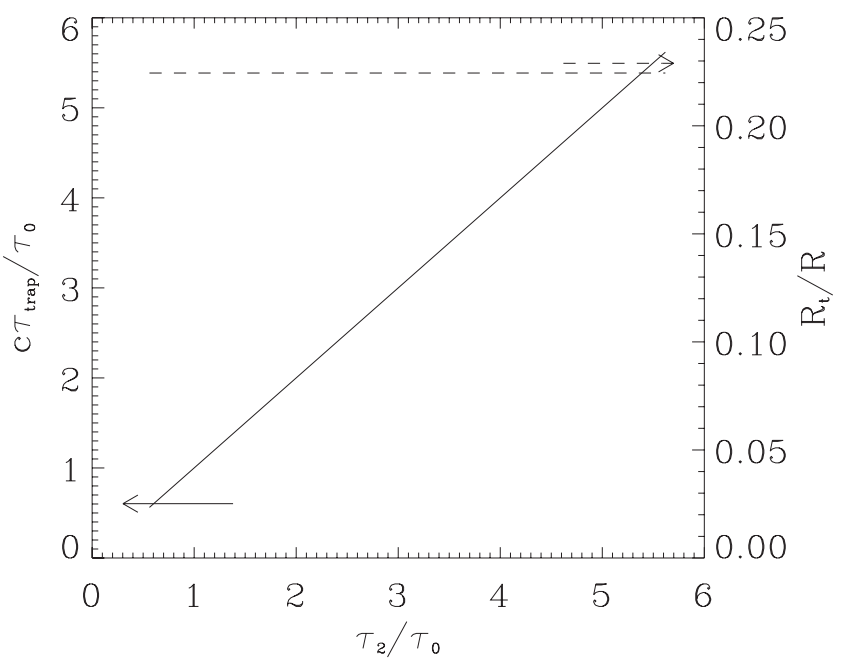

FIG. 7. Normalized trapping time and on-trap recombination coefficient as functions of characteristic time $\tau_{2}$ for spin-lattice coupling.

$$
\begin{aligned}
\operatorname{Im} \Pi_{\mathbf{q}, j}(\Omega)= & 2 \pi^{2} N(0) \Delta \int_{-\infty}^{\infty} d z \int_{-\Delta}^{\Delta} d z^{\prime}\left(\tanh \frac{z^{\prime}}{2 T}+\operatorname{coth} \frac{z}{2 T}\right) \\
& \times \delta\left(\Omega-z-z^{\prime}\right) \operatorname{Re} G_{0}(z)\left\{\left[\operatorname{Im} G\left(z^{\prime}\right)\right.\right. \\
& \left.+\frac{\Delta}{z} \operatorname{Im} F\left(z^{\prime}\right)\right]\left\langle\left|g_{\mathbf{q}, j}^{1}(\mathbf{p})\right|^{2}\right\rangle+S(S+1) \\
& \left.\times\left[\operatorname{Im} G\left(z^{\prime}\right)-\frac{\Delta}{z} \operatorname{Im} F\left(z^{\prime}\right)\right]\left\langle\left|g_{\mathbf{q}, j}^{2}(\mathbf{p})\right|^{2}\right\rangle\right\}, \quad(52)
\end{aligned}
$$

where $\operatorname{Re} G_{0}(z)=z / \sqrt{z^{2}-\Delta^{2}}$. The poles of the phonon Green's function are determined by

$$
\frac{\Omega^{2}-\omega_{\mathbf{q}, j}^{2}}{2 \omega_{\mathbf{q}, j}}-\Pi_{\mathbf{q}, j}(\Omega)=0 .
$$

Taking $\Omega=\omega_{\mathbf{q}, j}-i \gamma$ we obtain, after averaging over different phonon polarizations and directions of phonon wave vector,

$$
\begin{gathered}
\gamma=-\frac{\sum_{\mathbf{q}, j} \delta\left(\Omega-\omega_{\mathbf{q}, j}\right) \Pi_{\mathbf{q}, j}\left(\omega_{\mathbf{q}, j}\right)}{\sum_{\mathbf{q}, j} \delta\left(\Omega-\omega_{\mathbf{q}, j}\right)}= \\
-\frac{\sum_{\mathbf{q}, j} \delta\left(\Omega-\omega_{\mathbf{q}, j}\right) \Pi_{\mathbf{q}, j}\left(\omega_{\mathbf{q}, j}\right)}{N \Phi(\Omega)}
\end{gathered}
$$

where $N$ is ion density. Hence

$$
\begin{aligned}
\gamma(\Omega)= & -\frac{4 \pi N(0)}{N} \int_{-\infty}^{\infty} d z \int_{-\Delta}^{\Delta} d z^{\prime}\left(\tanh \frac{z^{\prime}}{2 T}+\operatorname{coth} \frac{z}{2 T}\right) \\
& \times \delta\left(\Omega-z-z^{\prime}\right) \operatorname{Re} G_{0}(z)\left\{\alpha _ { 1 } ^ { 2 } ( \Omega ) \left[\operatorname{Im} G\left(z^{\prime}\right)\right.\right. \\
& \left.+\frac{\Delta}{z} \operatorname{Im} F\left(z^{\prime}\right)\right]+S(S+1) \alpha_{2}^{2}(\Omega)\left[\operatorname{Im} G\left(z^{\prime}\right)\right.
\end{aligned}
$$




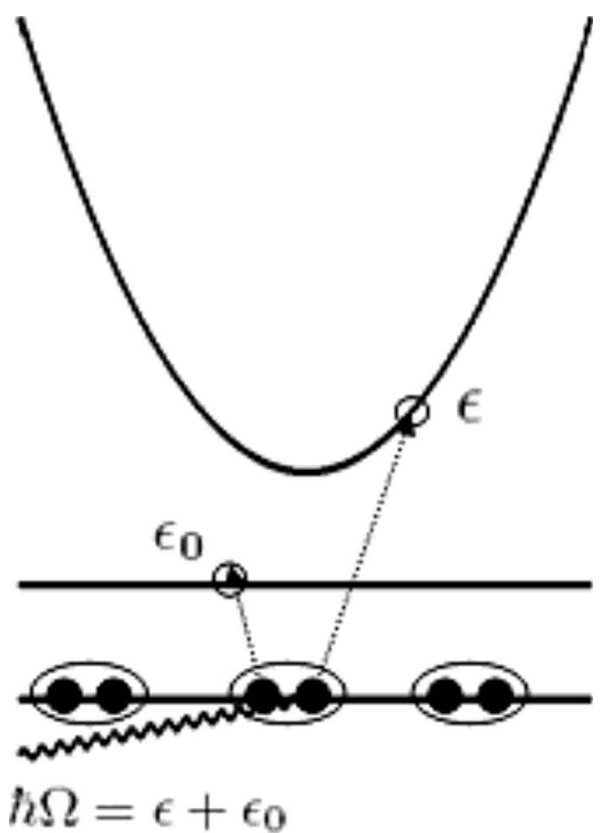

FIG. 8. Breaking of a Cooper pair by a phonon below the $2 \Delta$ threshold.

$$
\left.\left.-\frac{\Delta}{z} \operatorname{Im} F\left(z^{\prime}\right)\right]\right\} .
$$

It is important to note that the coherence factors in Eq. (55) are the same for pair breaking and recombination, and for trapping with phonon emission and detrapping with phonon absorption. Interference patterns are identical for both pairs of processes, and hence, coherence factors must be the same. This is a fundamental consequence of time-reversal symmetry and (as expected) is equally true for electron-phonon interactions in a superconductor involving only continuum ${ }^{15}$ and discrete states.

Calculating integrals in Eq. (55) for the dilute limit, we obtain

$$
\begin{aligned}
\gamma_{B}(\Omega)= & \frac{2 \pi N(0) \Delta c}{\hbar N} \frac{\left(1-y_{0}^{2}\right)^{3 / 2}}{1-y_{0}}\left[\alpha_{1}^{2}(\Omega)\left(\frac{\Omega / \Delta+1-y_{0}}{\Omega / \Delta-1-y_{0}}\right)^{1 / 2}\right. \\
& \left.+S(S+1) \alpha_{2}^{2}(\Omega)\left(\frac{\Omega / \Delta-1-y_{0}}{\Omega / \Delta+1-y_{0}}\right)^{1 / 2}\right]\left[1-f_{0}\left(\epsilon_{0}\right)\right. \\
& \left.-f_{0}\left(\Omega-\epsilon_{0}\right)\right]
\end{aligned}
$$

for the pair-breaking rate and

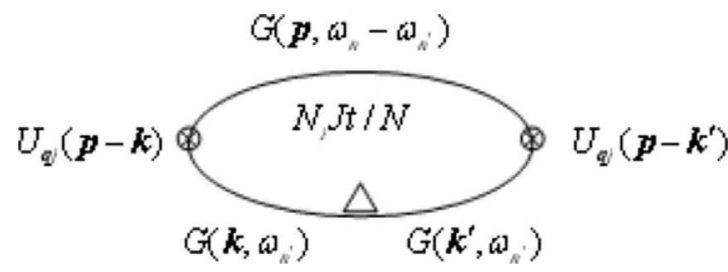

FIG. 9. Phonon self-energy.

$$
\begin{aligned}
\gamma_{p h s}(\Omega)= & \frac{2 \pi N(0)}{\hbar N} \frac{\left(1-y_{0}^{2}\right)^{3 / 2}}{1-y_{0}}\left[\alpha_{1}^{2}(\Omega)\left(\frac{\Omega / \Delta+1+y_{0}}{\Omega / \Delta-1+y_{0}}\right)^{1 / 2}\right. \\
& \left.+S(S+1) \alpha_{2}^{2}(\Omega)\left(\frac{\Omega / \Delta-1+y_{0}}{\Omega / \Delta+1+y_{0}}\right)^{1 / 2}\right] \\
& \times\left[f_{0}\left(\epsilon_{0}\right)-f_{0}\left(\Omega+\epsilon_{0}\right)\right]
\end{aligned}
$$

for phonon scattering.

\section{DISCUSSION}

\section{A. Coupling strength for continuum-bound-state transitions}

The existence of discrete intragap states opens up interaction channels which are not available in an ideal superconductor. The important question arises, therefore, as to whether there are circumstances under which the impurity effects may actually dominate intrinsic behavior. For example, the intrinsic recombination process should become increasingly inefficient as temperature decreases to a point when there are only a few thermally excited QPs left in the whole of a superconductor, yet it is often observed that QP lifetimes are finite even at the lowest temperatures. To determine whether the new interaction channels associated with the discrete levels due to magnetic impurities are capable of explaining these temperature anomalies, we must first evaluate the coupling strength for inelastic phonon-assisted transitions between continuum and discrete states.

The most convenient parameter for the strength of interaction is the electron-phonon coupling constant, which (in an ideal superconductor) is described by the Eliashberg function $\alpha^{2}(z) \Phi(z)$. In our earlier calculations of the effect of discrete states we introduced modified Eliashberg functions to discuss the interaction. We found that these depend on both the superconductor and the nature of the impurity itself. In order to evaluate the coupling strength for interactions involving discrete levels, we compare the two Eliashberg functions, one conventional and the other for the interaction involving the discrete state. Taking the ratio of two Eliashberg functions (31) and (32), after integration over angles, we obtain

$$
\frac{\alpha^{2}(z) \Phi(z)}{\alpha_{1,2}^{2}(z) \Phi(z)}=\frac{1}{2 \pi N(0) E_{F} \Delta a_{0}^{6}} \frac{\sum_{\mathbf{q}, j} 1 / v_{j} \delta\left(z-\omega_{\mathbf{q}, j}\right) \int\left(d O_{\mathbf{q}} / 4 \pi\right)(\mathbf{e} \cdot \mathbf{q} / q)^{2}\left|u_{e i}(\mathbf{q})\right|^{2}}{\sum_{\mathbf{q}, j} k_{F} / v_{j} q \delta\left(z-\omega_{\mathbf{q}, j}\right) \int\left(d O_{\mathbf{p}} / 4 \pi\right)\left\{\int\left(d O_{\mathbf{k}} / 4 \pi \mathbf{k}_{F}\right)\left[\left.\mathbf{e}_{\mathbf{q}, j} \cdot(\mathbf{p}-\mathbf{k}) u_{1,2}(\mathbf{p}-\mathbf{k})\right|_{k=k}\right]\right\}^{2}},
$$

where $v_{j}$ is the sound velocity for the $j$ branch. Evaluating $N(0)$ for the model of a spherical Fermi surface and approximating the integral over $\mathrm{d} O_{\mathbf{q}}$ by $\left|u_{e i}(\mathbf{q} \rightarrow 0)\right|^{2} \sim\left|u_{e i}(0)\right|^{2}$, we have 


$$
\frac{\alpha^{2}(z) \Phi(z)}{\alpha_{1,2}^{2}(z) \Phi(z)} \simeq \frac{1}{(2 \pi)^{3}} \frac{1}{\left(k_{F} a_{0}\right)^{6}} \frac{v_{F}}{v_{s}} \frac{z}{\Delta\left(d O_{\mathbf{p}} / 4 \pi\right)\left\{\int\left(d O_{\mathbf{k}} / 4 \pi k_{F}\right)\left[\left.\mathbf{e}_{\mathbf{q}, j} \cdot(\mathbf{p}-\mathbf{k}) u_{1,2}(\mathbf{p}-\mathbf{k})\right|_{k=k}\right]\right\}^{2}},
$$

where $v_{F}$ and $v_{s}$ are the Fermi velocity and mean sound velocity, respectively. Assuming that the impurity potentials are exponentially decaying functions with the radii $a_{1,2}$, we obtain the approximation $u_{1,2}(\mathbf{p}-\mathbf{k})=u_{1,2}(0) /[1+(\mathbf{p}$ $\left.-\mathbf{k})^{2} a_{1,2}^{2}\right]^{2}$. Here the form factor defines the interaction volume in momentum space. The remaining integrals in Eq. (59) are easily evaluated, leading to an estimate of the ratio of Eliashberg constants for the two types of interaction,

$$
\frac{\alpha^{2}(\Delta)}{\alpha_{1,2}^{2}(\Delta)} \simeq \frac{2}{\pi^{3}} \frac{v_{F}}{v_{s}} \frac{1}{\left(k_{F} a_{0}\right)^{2}}\left(\frac{a_{1,2}}{a_{0}}\right)^{4} \frac{\left|u_{e i}(0)\right|^{2}}{\left|u_{1,2}(0)\right|^{2}} .
$$

It follows from Eq. (60) that the characteristic ratio of coupling constants depends mainly on the sharpness of the scattering potentials. Thus for $a_{1,2} \simeq a_{0}$ the effect of coupling to an impurity is comparable to that of conventional electronphonon coupling due to the possibility of losing QP momentum by scattering on a sharply localized impurity potential. With increasing $a_{1,2}$ the interaction volume in momentum space rapidly shrinks because of its strong dependence on the form factor, so that coupling to an impurity state weakens. However in this situation the effects of intragap levels can be significant if the concentration of impurities is sufficiently high but still within the validity range of the dilute limit.

\section{B. Continuum-bound trapping}

In comparing the relative strengths of the intrinsic inelastic process and that leading to trapping at impurities, it is valuable first to contrast their dependence on QP energy. As seen from Eq. (45) for energies close to the gap edge, the dependence of the impurity trapping rate is weak but increases to a linear asymptotic dependence at high energies. The rate of spontaneous emission of phonons in intrinsic superconductors at $\epsilon \gg \Delta$ follows a cubic power of $\epsilon$, reflecting a quadratic density of states for phonons in the Eliashberg function $\alpha^{2}(z) \Phi(z) \propto z^{2}$. A lower power is found for transitions to bound states due to the reduction in the above exponential by one, leading to $\alpha_{1,2}^{2}(z) \Phi(z) \propto z$. This can be seen from the definition of coupling constants (30), which are inversely proportional to $\sqrt{\omega_{\mathbf{q}, j}}$. A further reduction in power exponent by unity arises from the fact that in an intrinsic superconductor, transitions may proceed into all states below the initial one with the integral resulting in an extra power in initial energy, which does not occur for transition into a discrete state. The smaller exponent for QP trapping on a discrete state has an important implication for nonequilibrium effects in multiple tunneling superconducting tunnel junctions (STJs). ${ }^{16,17}$

In order to estimate the order of magnitude of the continuum-bound trapping rate, we consider Ta as a typical superconductor. Assuming the presence of Kondo impurities with a discrete state residing deep inside the gap, we will take for an estimate $\epsilon_{0}=1 / 2 \Delta$. Using Eq. (45) we can estimate $\tau_{\text {trap }}=c^{-1} \tau_{2}$ for trapping from the edge of the gap $\epsilon$ $=\Delta$. Assuming also that the discrete state is strongly localized and taking $S(S+1)\left(a_{2} / a_{0}\right)^{4} \simeq 1$, we then have $\tau_{2} \simeq \tau_{0}$. For Ta $\tau_{0}=1.8 \mathrm{~ns}$ (Ref. 15$)$ so that $\tau_{\text {trap }} \sim 1.8 / c$ ns. The observed lifetimes in Ta, at such low temperatures that thermal recombination is absent, are typically several tens of microseconds ${ }^{11,18-23}$ leading to an estimate for $c$ of between $1 \times 10^{-5}$ and $1 \times 10^{-4}$ or a range of impurity concentration of 10-100 ppb. Thus even if the state were not strongly localized, for example, say $\left(a_{2} / a_{0}\right)^{4} \simeq 100$, in order to produce the observed QP lifetimes in Ta at low temperature, it would require a concentration of only 1-10 ppm. Such levels are still well below those usually regarded in content analyses as "trace" impurities.

\section{Continuum-bound recombination}

For reasons similar to those explained above, continuumbound recombination due to collisions with QPs occupying impurity levels has a different dependence on energy, being linear rather than quadratic, compared with intrinsic recombination. The ratio of the appropriate coefficients for the two types of recombination according to Eq. (51) is of the same order of magnitude as the ratio of respective Eliashberg constants (60), and hence, is close to unity for recombination on a strongly localized state.

Comparing the magnitudes of maximum recombination rates under quasiequilibrium conditions for the two different processes, we obtain

$$
\frac{R_{t} n_{t, T}}{R n_{T}}=c \sqrt{\frac{2 \Delta}{\pi T}} \exp \left(\frac{\Delta-\epsilon_{0}}{T}\right)\left(\frac{\Delta+\epsilon_{0}}{2 \Delta}\right) \frac{\alpha_{1}^{2}(\Delta)}{\alpha^{2}(\Delta)},
$$

where $n_{t, T}$ and $n_{T}$ are thermal distributions of trapped and mobile QPs. Hence, for even a small impurity density, recombination on the traps at low temperatures is a stronger process because of the presence of the exponential factor. The presence of this factor may significantly accelerate recombination at low temperatures in superconductors containing concentrations of magnetic impurities which are below trace levels. Moreover, the possible formation of an intragap band of bound states, and also of discrete bound states in the vicinity of the Fermi level, can significantly change the observed temperature dependence of recombination and thermalization rates.

An important consideration in discussing recombination in real samples is the phonon-bottleneck effect. A phonon which has been released in the recombination process must 


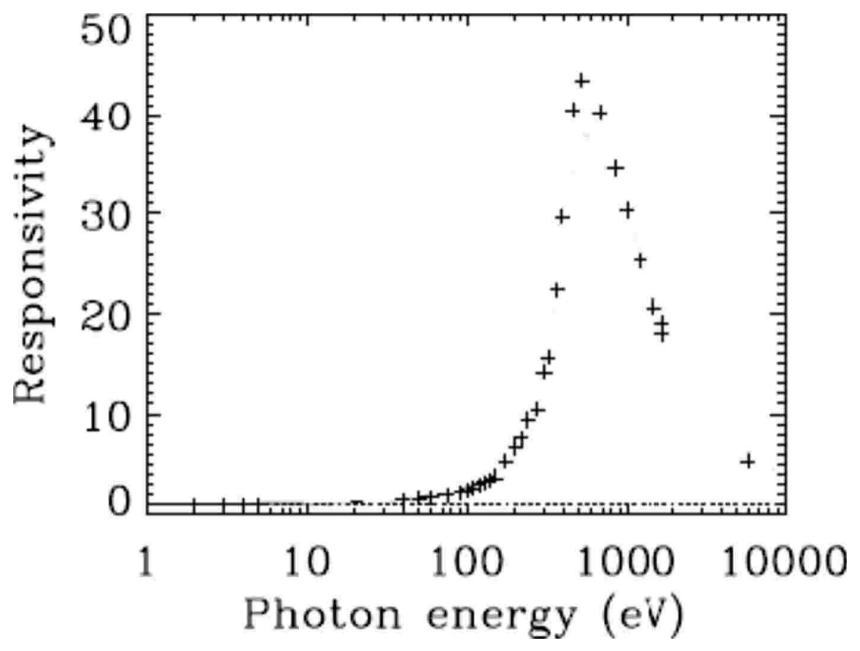

FIG. 10. Responsivity of a $\mathrm{Nb} / \mathrm{Al}_{2} \mathrm{O}_{3} / \mathrm{Nb}$ STJ as a function of photon energy.

be lost from the system to avoid breaking a further Cooper pair. When a QP with initial energy below $\Delta+\epsilon_{0}$ recombines with a second QP bound at an impurity with energy $\epsilon_{0}$, the emitted phonon has energy less than $2 \Delta$. This phonon can break a Cooper pair only if one of the resultant QPs occupies an impurity level, and therefore, has a much lower probability of pair breaking because of the low density of impurities. However, the decrease in pair-breaking efficiency for sub-2 $\Delta$ phonons may be partially compensated by their longer escape time, which occurs because phonons escape from an acoustically softer superconductor into a rigid dielectric substrate is constrained to lie within the critical escape cone defined by the critical incidence angle for the superconductor-substrate interface. ${ }^{16}$ Thus the process of phonon scattering from outside to inside the critical cone becomes crucial; in general, this conversion is less efficient for lower-energy phonons. Nevertheless despite the extra complications discussed above, impurity enhanced recombination is the principal recombination channel at the lowest temperatures because of the much higher occupation numbers for the impurity bound states.

\section{Analysis of existing experimental data}

Experimental data indicating the likely presence of such processes have been obtained by several groups. A crucial piece of evidence indicating the presence of local traps in superconductors is the strong dependence of the responsivity (charge output per unit deposited photon energy) of STJ photon detectors on the photon energy, ${ }^{24}$ as shown in Fig. 10 for a niobium STJ. The number of nonequilibrium quasiparticles generated by the absorption of a photon scales linearly with the photon energy $E$, while responsivity is directly proportional to QP lifetime. ${ }^{24}$ Thus the presence of a strong nonmonotonic dependence as shown in Fig. 10 is a direct evidence of quasiparticle trapping. The effect is most clearly seen at low densities of nonequilibrium QPs (small photon energies), since at the low temperature of the experiment $(\simeq 100 \mathrm{mK})$ most of the states on the traps are vacant and responsivity is at its lowest value. With increasing photon

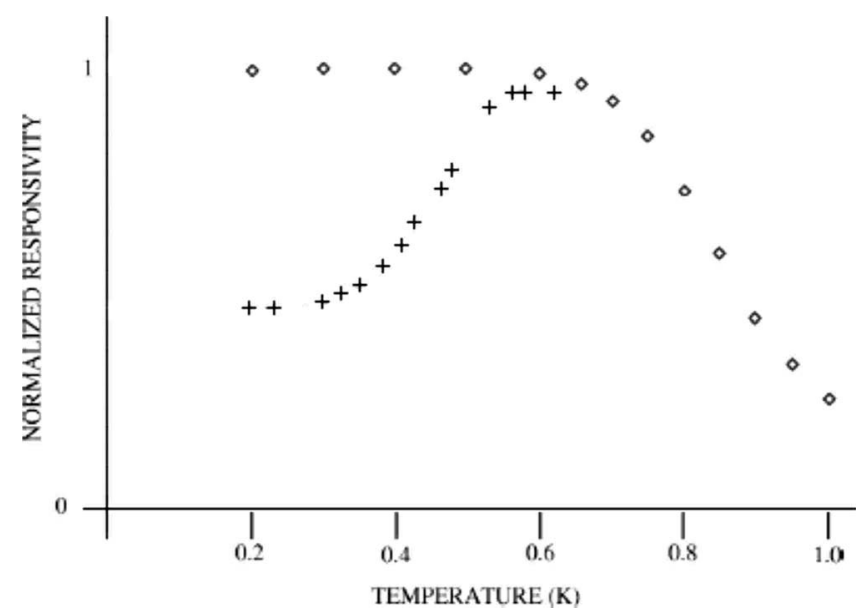

FIG. 11. Normalized responsivity of a symmetrical TaAl (30 $\mathrm{nm})$ STJ vs temperature: + are optical photons $(4.1 \mathrm{eV}) ; \diamond$ are x-ray $(5.9 \mathrm{keV})$ photons.

energy, responsivity starts rising as more QPs become trapped hence leaving fewer trap vacancies. With further increase in photon energy, and hence QP density, responsivity approaches a maximum as all traps become saturated. Finally at even higher QP densities the responsivity decreases because of recombination.

A second experiment highlighting the role of QP trapping is the measurement of the temperature dependence of responsivity, and therefore, of QP lifetime, ${ }^{11}$ as illustrated in Fig. 11 for X-ray and optical photons for a tantalum sample, with the data for both normalized to the value at $600 \mathrm{mK}$ showing the effects both of detrapping and thermal recombinations. The $\mathrm{x}$-ray responsivity does not fall off at low temperatures since the traps remain completely filled by the much greater number of generated QPs. Similar data were recently obtained by the kinetic inductance technique for tantalum and aluminum films on a different substrate. ${ }^{19}$ These are shown in Fig. 12. The inset of this figure shows that, contrary to the predictions of BCS theory for the ideal superconductor (dashed lines), the QP lifetime rises exponentially with decreasing tempera-

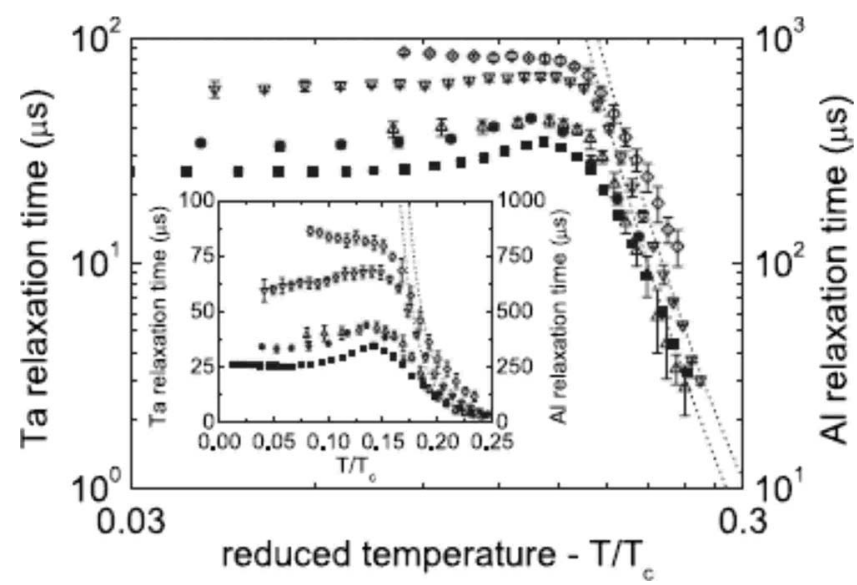

FIG. 12. The relaxation times as a function of reduced bath temperature for $150 \mathrm{~nm}$ Ta on $\mathrm{Si}$ (solid box, solid circle), $100 \mathrm{~nm} \mathrm{Al}$ on $\mathrm{Si}(\triangle), 250 \mathrm{~nm} \mathrm{Al}$ on $\mathrm{Si}(\nabla)$, and $250 \mathrm{~nm} \mathrm{Al}$ on sapphire $(\diamond)$ samples. The inset shows the same data on a linear scale. 
ture only at $T / T_{c} \geq 0.15$, remaining finite at low temperatures.

Values of QP lifetime obtained for a variety of different materials $^{20-23}$ at low temperatures are consistently, in some cases by orders of magnitude, shorter than can be explained simply by thermal recombination of nonequilibrium QPs (Ref. 15) and in addition are independent on temperature. It is likely that such relaxation process occurs through inelastic transitions with phonon emission as described earlier. As temperature increases, the QPs in bound states are activated into the continuum states and hence are able to contribute to the observed response. Ultimately, the effective QP lifetime increases with temperature until through the exponentially rising density of thermal excitations, thermal recombination becomes a dominant mechanism of QP relaxation.

Based on the theory developed in this paper quantitative analysis of such experimental data is now possible with the use of microscopic rates given above together with the rates for transitions in the continuum part of the spectrum. The main microscopic parameters are: concentration of magnetic impurities $c$, the discrete level energy $\epsilon_{0}$, the two characteristic times $\tau_{1}$ for the deformation-potential coupling and $\tau_{2}$ for spin-lattice coupling, which depend both on the host superconductor and the specific defect, and parameters describing the residual QP losses. None of the previous experimental data sets is sufficiently complete to determine all model parameters independently, and dedicated experiments are required in order to test the model in quantitative detail. The most important objective, however, is the identification of the defect itself which is responsible for breaking timereversal symmetry with the formation of a discrete intragap level.

\section{SUMMARY}

We have shown that the effect of small concentrations of magnetic impurities on transport properties of superconductors may be much greater than has previously been assumed. The localized QP states associated with discrete impurities facilitate trapping-related effects analogous to those occurring in semiconductors. It is likely that such processes are responsible for unexplained effects previously observed in samples containing such impurities only at trace level. The different mechanisms for inelastic scattering of the QPs are also relevant for electron decoherence in normal metals with Kondo impurities, which are currently the subject of great interest. $^{25,26}$

\section{ACKNOWLEDGMENTS}

We acknowledge valuable discussions with T. M. Klapwijk, R. Barends, and J. R. Gao.
${ }^{1}$ A. A. Abrikosov and L. P. Gor'kov, Sov. Phys. JETP 12, 1243 (1961).

${ }^{2}$ L. Yu, Acta Phys. Sin. 21, 75 (1965).

${ }^{3}$ H. Shiba, Prog. Theor. Phys. 40, 435 (1968).

${ }^{4}$ H. Shiba, Prog. Theor. Phys. 50, 50 (1973).

${ }^{5}$ A. I. Rusinov, JETP Lett. 9, 85 (1969).

${ }^{6}$ A. I. Rusinov, Sov. Phys. JETP 29, 1101 (1969).

${ }^{7}$ E. Müller-Hartmann and J. Zittartz, Phys. Rev. Lett. 26, 428 (1971).

${ }^{8}$ J. Zittartz and E. Müller-Hartmann, Z. Phys. 232, 11 (1970).

${ }^{9}$ A. Yazdani, B. A. Jones, C. P. Lutz, M. F. Crommie, and D. M. Eigler, Science 275, 1767 (1997).

${ }^{10}$ A. V. Balatsky, I. Vechter, and Jian-Xin Zhy, Rev. Mod. Phys. 78, 373 (2006).

${ }^{11}$ A. G. Kozorezov, J. K. Wigmore, A. Peacock, A. Poelaert, P. Verhoeve, R. den Hartog, and G. Brammertz, Appl. Phys. Lett. 78, 3654 (2001).

${ }^{12}$ G. M. Eliashberg, Sov. Phys. JETP 11, 696 (1960).

${ }^{13}$ S. V. Vonsovsky, Yu. A. Izyumov, and E. Z. Kumaev, Superconductivity of Transition Metals (Springer-Verlag, Berlin, 1982).

${ }^{14}$ M. Tinkham, Introduction to Superconductivity (McGraw-Hill, New York, 1996).

${ }^{15}$ S. B. Kaplan, C. C. Chi, D. N. Langenberg, J. J. Chang, S. Yafarey, and D. Scalapino, Phys. Rev. B 14, 4854 (1976).

${ }^{16}$ A. G. Kozorezov, R. A. Hijmering, G. Brammertz, J. K. Wigmore, A. Peacock, D. Martin, P. Verhoeve, A. A. Golubov, and
H. Rogalla, Phys. Rev. B 77, 014501 (2008).

${ }^{17}$ R. Gross and D. Koelle, Rep. Prog. Phys. 57, 651 (1994).

${ }^{18}$ P. Verhoeve, R. den Hartog, A. G. Kozorezov, D. Martin, A. van Dordrecht, J. K. Wigmore, and A. Peacock, J. Appl. Phys. 92, 6072 (2002).

${ }^{19}$ R. Barends, J. J. A. Baselmans, S. J. C. Yates, J. R. Gao, J. N. Hovenier, and T. M. Klapwijk, Phys. Rev. Lett. 100, 257002 (2008).

${ }^{20}$ Th. Nussbaumer, Ph. Lerch, E. Kirk, A. Zehnder, R. Fuchslin, P. F. Meier, and H. R. Ott, Phys. Rev. B 61, 9719 (2000).

${ }^{21}$ L. Li, L. Frunzio, C. M. Wilson, and D. E. Prober, J. Appl. Phys. 93, 1137 (2003).

${ }^{22}$ B. A. Mazin, B. Bumble, P. K. Day, M. E. Eckart, S. Golwala, J. Zmuidzinas, and F. E. Harrison, Appl. Phys. Lett. 89, 222507 (2006).

${ }^{23}$ J. P. Porst, C. Höhn, D. Haug, R. Weldle, G. M. Seidel, L. Gastaldo, A. Fleischmann, and C. Enss, J. Low Temp. Phys. 151, 436 (2008).

${ }^{24}$ A. Poelaert, A. G. Kozorezov, A. Peacock, and J. K. Wigmore, Phys. Rev. Lett. 82, 1257 (1999).

${ }^{25}$ Gassem M. Alzoubi and Norman O. Birge, Phys. Rev. Lett. 97, 226803 (2006).

${ }^{26}$ F. Mallet, J. Ericsson, D. Mailly, S. Unlubayir, D. Reuter, A. Melnikov, A. D. Wieck, T. Micklitz, A. Rosch, T. A. Costi, L. Saminadayar, and C. Bäuerle, Phys. Rev. Lett. 97, 226804 (2006). 